\title{
The digital agenda of virtual currencies: Can BitCoin become a global currency?
}

\author{
Pavel Ciaian ${ }^{1} \cdot$ Miroslava Rajcaniova ${ }^{2}$. \\ d'Artis Kancs'
}

Received: 21 August 2014/Revised: 30 June 2015/ Accepted: 4 January 2016/

Published online: 2 February 2016

(C) The Author(s) 2016. This article is published with open access at Springerlink.com

\begin{abstract}
This paper identifies and analyzes BitCoin features which may facilitate BitCoin to become a global currency, as well as characteristics which may impede the use of BitCoin as a medium of exchange, a unit of account and a store of value, and compares BitCoin with standard currencies with respect to the main functions of money. Among all analyzed BitCoin features, the extreme price volatility stands out most clearly compared to standard currencies. In order to understand the reasons for such extreme price volatility, we attempt to identify drivers of BitCoin price formation and estimate their importance econometrically. We apply time-series analytical mechanisms to daily data for the 2009-2014 period. Our estimation results suggest that BitCoin attractiveness indicators are the strongest drivers of BitCoin price followed by market forces. In contrast, macro-financial developments do not determine BitCoin price in the long-run. Our findings suggest that as long as BitCoin price will be mainly driven by speculative investments, BitCoin will not be able to compete with standard currencies.
\end{abstract}

Keywords BitCoin - Virtual currency · Exchange rate - Supply and demand . Financial indicators · Attention-driven investment

\section{Introduction}

A wide range of virtual currencies have emerged during the last decade, such as BitCoin, LiteCoin, PeerCoin, AuroraCoin, DogeCoin and Ripple. The most successful among them is BitCoin, both in terms of its impressive growth in the

Pavel Ciaian

pavel.ciaian@ec.europa.eu

1 European Commission, DG Joint Research Centre, C/Inca Garcilaso, 3, 41092 Seville, Spain

2 Slovak University of Agriculture (SUA), Nitra, Slovakia 
number of currency users and popularity by retailers. Since its introduction in 2009 , BitCoin has been characterized also by a phenomenal increase in the number of transactions and market capitalization, which surpassed 5 billion US dollar in 2015 and since then has recorded further growth.

In the public media and in scientific community there is ongoing a lively debate on wheather BitCoin can actually function as a substitute for standard currencies such as US dollar, Euro or Yen. There are two competing views in the literature on whether BitCoin fulfils the three criteria of a currency (a medium of exchange, a unit of account, and a store of value) (Mankiw 2007). One part of the literature argues that BitCoin does not behave as a real currency, but rather resembles speculative investments (e.g. Velde 2013; Hanley 2014; Yermack 2014; Williams 2014). Other line of research, stresses the positive aspects of BitCoin and perceives it as a global virtual currency with strong future potential (e.g. Plassaras 2013; Satran 2013; Luther and White 2014; Folkinshteyn et al. 2015). Also from a legal point of view, there is no global agreement on the status of BitCoin, as there are no international laws regulating BitCoin. Each country regards BitCoin differently and regulations are constantly evolving. For example, the Internal Revenue Service in the US has assigned BitCoin as barter on the grounds of the market-orientated approach. Finland considers BitCoin as priced commodity. Germany has recognized it as private currency. Yet other EU Member States by and large are in the wait-andsee stage. On the other hand, institutions such as European Central Bank (ECB) and European Banking Authority (EBA) define BitCoin as virtual currency.

In light of these and other open questions about BitCoin, in April 2015 the European Commission organized a Virtual Currencies Conference, ${ }^{1}$ where among others the potential and challenges of BitCoin to become a global currency were discussed. The Virtual Currencies Conference acknowledged the need for further research to better understand the socio-economic and monetary aspects of the virtual currency ecosystem.

In order to shed light on these and related questions, the present paper attempts to identify and analyze BitCoin features which may facilitate BitCoin to become a global currency, as well as characteristics which may impede the use of BitCoin as a medium of exchange, a unit of account, or a store of value. Among the BitCoin features, which may facilitate its use as a currency, we have identified low transaction costs, high anonymity and privacy, learning spillover effects, infinite divisibility and no inflationary pressures. Among the BitCoin features, which may impede its use as a currency include the absence of a legal tender attribute, difficulty to procure BitCoins, relatively high fixed costs of adoption, dependence on network externalities, absence of an institution enforcing dispute resolution, absence of BitCoin denominated credits, deflationary pressure, extremely high price volatility, and issues with cyber security.

Our second contribution to the literature is to undertake a comparative analysis of the identified BitCoin features with traditional currencies and their possible impact on BitCoin functions as a currency. Our analysis builds on previous studies, which have identified several advantages of BitCoin over traditional currencies. First, as

\footnotetext{
${ }^{1}$ http://ec.europa.eu/digital-agenda/en/news/blockchain-and-digital-currencies-workshop.
} 
noted by EPRS (2014), EBA (2014), and Folkinshteyn et al. (2015), BitCoin has no physical representation, such as paper bills or metal coins, which saves costs related to the production, transportation, and handling of physical currency. These costs can be substantial for standard currencies. Similarly, an advantage of BitCoin is that it is more convenient than standard payment mechanisms for small-value purchases, as it allows for money transfers at low costs and relatively fast (Hayes et al. 1996). As noted by Folkinshteyn et al. (2015), the advantages of low transaction fees and short execution time make BitCoin an ideal medium of exchange. In contrast, Grinberg (2011) opposes this view and argues that the response of the traditional e-commerce sector to the competitive pressure from BitCoin may induce them to reduce their transaction costs and thus offset the advantage of BitCoin, while providing also other benefits such as higher security. Second, an additional advantage of BitCoin, compared to standard currencies, is that it may reduce opportunities for theft, such as bank robbery. BitCoin could help curtail vandalism of vending machines, public phones, etc., because there would be no cash to steal. Similarly, businesses who handle cash, such as taxi drivers and small shops, could be much less vulnerable to robbery, if they would use BitCoin. Finally, BitCoin is a global currency, implying that there are no transaction costs related to currency exchange.

On the other hand, BitCoin faces several challenges compared to standard currencies. One of the main challenges of BitCoin is its security (Moore and Christin 2013; Böhme et al. 2015; Yermack 2014). Given that BitCoin transactions take place exclusively over the internet, cyber-security is its main threat. Particularly vulnerable to cyber-attacks are large holdings of BitCoins as well as BitCoin exchanges. The security problem is largely attributed to the lack of an oversight institution that would ensure security of BitCoin transactions and BitCoin system (ECB 2012; Plassaras 2013; Moore and Christin 2013). Second, the use of a new currency, such as BitCoin, requires an initial investment for both businesses and consumers (Velde 2013). This includes costs linked to getting acquainted with BitCoin system in general and to the adoption of the payment technology in particular. For example, BitCoin requires the use of electronic devices including specific software. Similarly, BitCoin may also suffer from information asymmetry, as its system is relatively complex and therefore may not be easily understood by all potential users (ECB 2012). Third, Yermack (2014) argues that BitCoin may fail to become a global medium of exchange, as it is used in too few exchanges of goods and services; it has a negligible market presence globally. Currently, there are around 0.11 million BitCoin transactions executed per day (BlockChain 2015) and most of these BitCoin transactions involve transfers between speculative investors. BitCoin use for purchases of goods and services is only minor-20\% of all BitCoin transactions (Yermack 2014). For example, if compared to 295 million conventional payments and terminal transactions per day done in Europe alone, the market share of BitCoin appears to be minuscule (EBA 2014). Fourth, Yermack (2014) argues that BitCoin is ineffective as a tool of risk management of price volatility as it is uncorrelated with major world currencies (i.e. US dollar, Euro, Yen, British Pound) or gold price, which is a common use of currencies by businesses for hedging the risks associated with currency volatility. Macroeconomic changes that cause adjustment in exchange rates of currencies are not reflected in any way in BitCoin 
price movements implying that its price volatility cannot be easily hedged. Finally, BitCoin faces the problem of network externalities in its adoption (Gowrisankaran and Stavins 1999), as its benefit in exchange is positively correlated with the number of users (Plassaras 2013; ECB 2012).

Among all BitCoin features that we have identified and analyzed, eventually, price volatility is the one with the largest differences compared to standard currencies, such as US dollar, Euro, Yen, British Pound. As shown in Fig. 1, since its introduction in 2009, BitCoin price has shown extremely high volatility; it has increased from zero value at the time of its inception in 2009 to around $\$ 1100$ at the end of 2013 when it reached the peak followed by a decline to around \$225 (see Fig. 1). For comparison, the fluctuation of exchange rate between US dollar and Euro has not exceeded a $\pm 20 \%$ bound in the same 6 year period (see Fig. 1). One consequence of such extreme price volatility is a threat for BitCoin being accepted as a global currency, as it may fail to accurately convey relative prices of goods and services in the economy and may generate uncertainties to its holders due to its inability to preserve stable value over time. Indeed, a desirable property of any monetary mean is that it holds its value over short-to-medium periods of time, in order not to create distortion when used as a medium of exchange in transactions, a unit of account, and a store of value. Large price movements alter the purchasing power by causing risk and costs to firms and consumers using BitCoin. Given that BitCoin is a relatively new currency, its price formation is not well understood yet, as there are only few studies on BitCoin price formation available in the literature (e.g. Buchholz et al. 2012; Kristoufek 2013; van Wijk 2013; Bouoiyour and Selmi 2015).

In order to better understand the reasons for such extremely high price volatility, we attempt to identify drivers of BitCoin price and estimate their importance econometrically. This is our third contribution to the literature. Previous studies (e.g. Buchholz et al. 2012; Kristoufek 2013; van Wijk 2013; Bouoiyour and Selmi 2015) suggest three types of drivers determining BitCoin price development: (1) market forces of BitCoin supply and demand (2) BitCoin attractiveness, and (3) global macroeconomic and financial developments. We apply time-series analytical

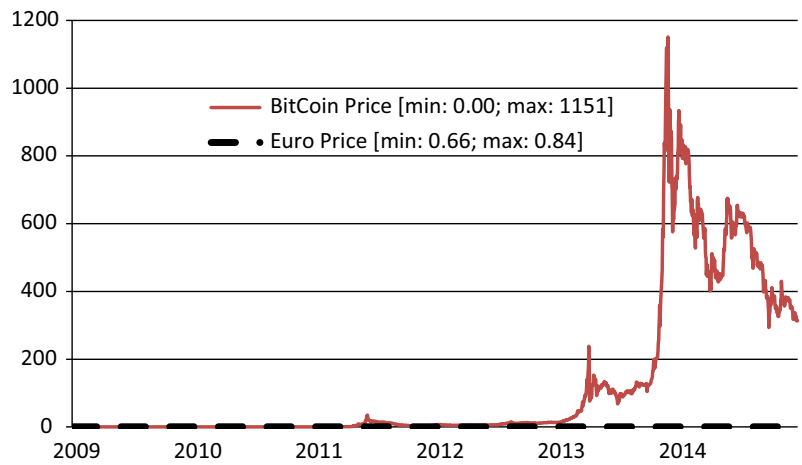

Fig. 1 BitCoin and Euro price development in USD, 2009-2011. Source: BlockChain and Oanda 
mechanisms to these drivers using daily data for the 2009-2014 period. Our estimation results suggest that BitCoin attractiveness variables are most important drivers of BitCoin price followed by market forces. Our estimates do not support previous findings that macro-financial developments are driving BitCoin price in the long-run.

Generally, our findings suggest that as long as BitCoin price will be mainly driven by speculative investments, BitCoin will not be able to compete with standard currencies.

The rest of the paper is structured as follows. Section 2 provides background information about BitCoin, which is a relatively new virtual currency. In the context of the three currency criteria, the key features are discussed and contrasted with standard currencies to analyze whether BitCoin fulfills the main functions of money. Section 3 identifies the main factors affecting BitCoin price based on previous studies; outlines the econometric approach - a Vector Auto Regressive (VAR) model - and specifies the estimated model. It also details the data sources used in the empirical analysis, the construction of the estimable model's variables, and discusses the estimation results. The final section concludes.

\section{Is BitCoin a currency?}

\subsection{Background of BitCoin}

Currency, or money in general, is typically defined as having three main functions: a medium of exchange, a unit of account, and a store of value (Mankiw 2007). Among different types of currencies, virtual currencies are clearly distinguished from fiat currencies (i.e. "real currency," "real money," "national currency", or "standard currency"), which is in coin and paper format, designated as legal tender, regulated by central bank and used and accepted as a medium of exchange in the issuing country (Table 1) (FATF 2014). According to the definition proposed by the ECB (2012) "a virtual currency is a type of unregulated, digital money, which is issued and usually controlled by its developers, and used and accepted among the members of a specific virtual community". Similarly, according to the EBA (2014), "[virtual currencies] are defined as a digital representation of value that is neither issued by a central bank or public authority nor necessarily attached to a [conventional

Table 1 Type of currencies

\begin{tabular}{lll}
\hline & Money (currency) format & \\
\cline { 2 - 3 } & Physical & Digital \\
\hline $\begin{array}{l}\text { Legal status } \\
\text { Unregulated }\end{array}$ & Certain types of local currencies & Virtual currency \\
Regulated & Banknotes and coins & E-money \\
& & Commercial bank money (deposits) \\
\hline
\end{tabular}

Source ECB (2012) 
currency], but is used by natural or legal persons as a means of exchange and can be transferred, stored or traded electronically." 2

The most popular virtual currency is BitCoin, created by the Japanese programmer Satoshi Nakamoto in 2009 (Nakamoto 2009). It was the first open source virtual currency, as BitCoin is managed by an open source software algorithm that uses the global internet network both to create BitCoins as well as to record and verify its transactions. Being a cryptocurrency, BitCoin uses the principles of cryptography to control the creation and exchange of BitCoins. BitCoins can be stored in local wallets (e.g. personal computer, smartphone) using an open-source software or in an online wallet (Brito and Castillo 2013; Murphy 2013; CoinDesk 2015b).

Compared to standard fiat currencies, such as US dollars or Euro, a distinguishing feature of BitCoin is that the quantity of units in circulation is not controlled by a person, group, company, central authority, or government, but by a software algorithm. BitCoins are created in a 'mining' process, in which computer network participants, i.e. users who provide their computing power, verify and record payments into a public ledger called BlockChain. In return for this service they receive transaction fees and newly minted BitCoins. A fixed amount of BitCoins is issued at a constant a priori defined and publicly known rate, according to which the stock of BitCoins increases at a decreasing rate. In 2140 the growth rate of BitCoin will converge to zero, when the maximum amount of BitCoins in circulation will reach 21 million units; according to the current algorithm it will not change after 2140.

BitCoin does not have physical representation. Instead, it is stored either on electronic devices (e.g. personal computer, mobile, tablet) or entrusted to an online service and is transferred via the internet. BitCoins can be spent on both goods and services, if accepted by the retailer. Users interact with each other directly and anonymously, without third-party intervention. There is neither a central clearing house nor any other intermediary institution involved in the transactions such as central bank or government agency (Table 1). Currently, BitCoins can be acquired either (1) by exchanging them for standard money (e.g. US dollar, Euro) on a BitCoin exchange or from a BitCoin dealer, (2) by obtaining them from sales of goods or services denominated in BitCoin, or (3) through a mining process (Plassaras 2013; CoinDesk 2015c).

BitCoins can be used to buy goods or services worldwide, provided that the transaction partner accepts BitCoin as a mean of payment. A transaction implies that the present owner of BitCoins transfers the ownership of a certain amount of BitCoins to a different market participant in exchange for other currencies, goods or services. A continuously growing number of companies accept BitCoins as payments for their goods and services, at the beginning of 2015 there were more than 100,000 venues accepting BitCoins (CoinDesk 2015a; Cuthbertson 2015).

\footnotetext{
${ }^{2}$ Note that virtual currencies must be distinguished from electronic money. The key distinguishing feature of electronic money is that their link with traditional money is preserved and have the same unit of account as well as they have legal foundation and are regulated. This is not the case of virtual currencies including BitCoin (ECB 2012).
} 
There are two competing views in the literature on whether BitCoin fulfils the three criteria of a currency (a medium of exchange, a unit of account, and a store of value). One part of the literature argues that BitCoin does not behave largely like a real currency, as it does not fulfil the main functions of a currency, but rather serves as a vehicle for speculative investments (Velde 2013; Hanley 2014; Yermack 2014; Williams 2014). Other line of research stresses the potential of BitCoin and perceives it as a global currency with strong future potential (e.g. Plassaras 2013; Satran 2013; Luther and White 2014; Folkinshteyn et al. 2015). In the following we attempt to identify and analyze the most important advantages and disadvantages of BitCoin in the context of the three currency functions.

\subsection{Medium of exchange}

One of the main functions of any currency is to intermediate the exchange of goods and services. We identify several features of BitCoin as a medium of exchange, which differ substantially from traditional currencies: transaction costs, anonymity and transparency, legal tender, fixed costs, network externalities, dispute resolution, and credit market (see Table 2 for summary).

\subsubsection{Transaction costs}

An appealing feature of BitCoin when used as a medium of exchange is its comparative advantage in transaction costs relative to standard currencies, as BitCoin transaction fees are considerably lower than comparable costs of traditional means of payment (e.g. payment cards or bank transfers). BitCoin transaction fees cover only the costs of maintaining the system (i.e. clearing system) paid to miners; there are no costs linked to third-party intermediaries, such as a regulatory authority, that would perform validation, storage or security functions; the BitCoin system (maintained by miners) is financed through transaction fees and newly issued

Table 2 Currency characteristics of BitCoin with respect to standard currencies

\begin{tabular}{lll}
\hline & Advantage of BitCoin & Disadvantage of BitCoin \\
\hline Medium of exchange & $\begin{array}{l}\text { Transaction costs } \\
\text { Anonymity and privacy }\end{array}$ & \\
& Learning spillover effect & Fot legal tender and difficulty to procure BitCoins \\
& & Network externalities \\
& & Dispute resolution not available \\
& & Absence of BitCoin denominated credit \\
Unit of account & Divisibility & Relative price comparability problem \\
Store of value & Price volatility \\
& Non-inflationary supply & Deflationary pressure \\
& Cyber security \\
\hline
\end{tabular}

Source Authors' presentation 
BitCoins. ${ }^{3}$ Transaction costs of standard currencies are considerably higher as they need to cover all costs of intermediaries. Besides the costs of clearing system, the fees charged for standard money transfers need to also offset the cost of storage, authentication, transport, security, etc. (Šurda 2012; EBA 2014). Average transaction fees per transaction for BitCoin transfers are between 0 and $1 \%$, while traditional online payment systems charge fees between 2 and $5 \%$ or even more (EPRS 2014; EBA 2014; Folkinshteyn, Lennon and Reilly 2015). Estimates for the US dollar show that these costs (costs of processing and accounting of money, storage, transport, and security) account to as much as $\$ 60$ billion annually (ca. $4.4 \%$ of the US dollar value). ${ }^{4}$ Further, BitCoin offers faster transaction execution than traditional online payment systems. The total processing time of BitCoin transfers is between 10 and 60 min (EBA 2014).

We may conclude that comparably low transaction and maintenance costs together with fast execution of transactions may facilitate the acceptance and use of BitCoin as a currency (Table 2).

\subsubsection{Anonymity and transparency}

An area, where BitCoin has a relatively high appeal, involves exchanges linked to illegal and criminal activities (e.g. money laundering; narcotic trade, tax evasion). While this was not the intent of BitCoin creators, it is rather the way individuals choose to use it, as certain BitCoin characteristics give its predisposition to be advantageous in such activities. For example, BitCoin payment transactions are anonymous and do not require the provision of personal identity information. Further, BitCoin allows for easy international transfers (e.g. money laundering) without supervision, as BitCoin transactions are peer-to-peer and require just internet access. With its infrastructure being spread across the globe, it is difficult to intercept individual transactions (Bryans 2014; EBA 2014). These features grant BitCoin a rather high predisposition to be used in illegal activities. For example, the internet portal Silk Road, created in January 2011, provided online marketplace for the sale of illegal narcotics and weapons using BitCoins for payment, accounting for as much as half of all BitCoin transactions. Silk Road was shut down following an FBI investigation in 2013. Often it is argued that this event generated benefit to BitCoin by boosting its popularity (EBA 2014; Yermack 2014).

Gambling is other area where BitCoin appears to be growing as a medium of exchange, as it protects costumer privacy and allows receiving funds from consumers not being able to use other payment methods. The most popular BitCoin denominated online gambling site is Satoshi Dice operating since 2012 (Böhme et al. 2015). Generally, there is a growing number of online gambling sites, the BitCoin Wiki (2015) lists around 100 BitCoin based casinos, poker sites, bingo games, betting services and lotteries.

\footnotetext{
${ }^{3}$ Given that newly issued BitCoins decrease over time, miners will have to rely more on transaction fees to recoup their investment in mining which may lead to higher transaction fees in the future (EBA 2014). For a theoretical analysis of the economics of BitCoin transaction fees see Kroll et al. (2013) and Houy (2014).

4 There was approximately $\$ 1.37$ trillion in circulation as of June, 2015 (Federal Reserve System 2015).
} 
The BitCoin platform is transparent and public, meaning that anyone is able to follow the chain of transaction. All BitCoin payments have a traceable history that can be viewed by anyone. A single anonymity breach can uncover a user's entire BitCoin transaction history. For example, the knowledge of the identity of any user from any transaction (e.g. obtained from the mailing address used for delivery of purchased goods with BitCoin, or from the bank account used to purchase BitCoin) allow to track that user's transactions backward and forward through the BlockChain history. Hence, BitCoin system is often referred as pseudonymous in the sense that full history of all transactions and every BitCoin is preserved on the publicly ledger. However, several options are available to overcome this anonymity problem and make transactions non-traceable, for example, by using new addresses for each payment received, using change addresses when sending payments, or using BitCoin mixer services to break the link between an user and its BitCoins (Bitcoinhelp 2014; Crawford 2014; BitCoin 2015; Böhme et al. 2015).

We may conclude that relatively high anonymity of BitCoin users and transparency of BitCoin transactions may facilitate the popularity and use of BitCoin as a currency (Table 2).

\subsubsection{Legal tender}

One distinguishing feature of BitCoin is that it is not a legal tender (as opposed to standard currencies which would imply its mandatory acceptance in exchanges). Private or public businesses are not legally obliged to accept BitCoin as a payment form for goods and services they trade. The use of BitCoin as a medium of exchange is thus fully dependent on its voluntary adoption by market participants (EBA 2014).

According to Yermack (2014), an additional obstacle for BitCoin to become a widely used medium of exchange, arises from the difficulty of procuring new BitCoins. BitCoins can be obtained only from online exchanges or dealers (except for successful BitCoin miners).

We may conclude that, generally, the absence of legal tender may impede BitCoin use as a currency, because any business can decide individually on the acceptance/not acceptance of BitCoin (Table 2).

\subsubsection{Fixed costs}

The use of a new currency, such as BitCoin, requires an initial investment for both businesses and consumers. This includes costs linked to getting acquainted with the BitCoin system in general and to the adoption of the payment technology in particular. For example, BitCoin requires the use of electronic devices including specific software. ${ }^{5}$

\footnotetext{
5 Access to the BitCoin network requires downloading a BitCoin software on personal computer and joining the BitCoin network, which allows users to engage in operations, and update and verify transactions.
} 
In addition, a relatively high level of computer knowledge is required for understanding and using BitCoins, which may represent a barrier to many potential users and may constraint its wider adoption (i.e. information asymmetry problem). BitCoin is based on a complex code, which is understood by only a few persons and is controlled by even fewer individuals (programmers), who oversee the whole system without accountability, arbitration or recourse (Velde 2013; Yermack 2014).

On the other hand, BitCoin use may increase users' interaction with virtual currencies and thus may have learning spillover effects by helping to improve their skills and knowledge in virtual transactions and online financial applications (Hayes et al. 1996; Berentsten 1998; Plassaras 2013).

We may conclude that in presence of high fixed costs, the popularity and use of BitCoin as a currency may be seriously impeded at least in the short-run, as currently the technical knowledge about BitCoin is still rather low in society. However, BitCoin may generate some additional benefits to its users through learning spillover effects (Table 2).

\subsubsection{Network externalities}

The incentives for market participants to use BitCoin depend on the number of existing users. That is, the benefit of making the investment and using it in exchanges depends on the number of other suppliers and consumers of goods and services using BitCoin. If only a few businesses accept BitCoins, there are little incentives for consumers to acquire them. On the other hand, if only a few consumers use BitCoins, businesses have little incentives to invest into the equipment for processing BitCoin payments for their goods and services. This is a well-known problem of network externalities (Gowrisankaran and Stavins 1999). Hence, one of the main BitCoin challenges in becoming a global currency is to convince users to use it in their purchases and businesses to accept it as payment form for their goods and services (Berentsten 1998; ECB 2012; Plassaras 2013).

Yermack (2014) argues that BitCoin largely fails to be a global medium of exchange as it is used to a limited extent to intermediate the exchange of goods and services. Currently there are around 0.11 million BitCoin transactions executed per day (BlockChain 2015) which is insignificant compared, for example, to 295 million conventional payments and terminal transactions per day done in Europe alone (EBA 2014). According to BlockChain (2015), the use of BitCoin as a medium of exchange is continuously increasing over time as more and more businesses tend to accept it as a form of payment. Hence, despite the rather low BitCoin penetration in the global exchanges currently may not be an obstacle for BitCoin to become a global medium of exchange in future. First, given that BitCoin is a relatively new currency, it may take some time till it gets adopted in a wider context. Second, there exist examples of small countries with own currencies (e.g. Fiji) which intermediate the exchange of goods and services within the national boundaries but have only a minor share globally.

We may conclude that the issue of network externalities may impede growth of BitCoin use as a currency in the short-run. However, these issues may become less relevant in future, as BitCoin use continuously increases (Table 2). 


\subsubsection{Dispute resolution}

The use of BitCoin in market exchanges bears a certain risk, because of the absence of any protection against disputes between parties involved in the exchange. Once a BitCoin transaction is realized, it is irreversible and cannot be disputed. There is no centralized mechanism available to revert an erroneous transaction or to handle the disputes with the aim to provide protection against human errors of fraud that may occur in exchanges (e.g. protection against disputes over non-fulfillment of contract). Currently, the correction of an erroneous transaction is possible only through a voluntary agreement of the parties involved in the exchange (EBA 2014; Böhme et al. 2015).

We may conclude that the absence of an institution regulating and enforcing BitCoin related disputes, the popularity and use of BitCoin as a currency may be impeded, particularly for risk-averse market participants (Table 2).

\subsubsection{Credit market}

Another obstacle for BitCoin to become a widely used medium of exchange may be that BitCoin cannot be used to take loans because, under the current system, every loan would need to be made in BitCoin. Whereas the basis of standard banking system is fractional-reserve, which determine how much new money can be created through loans, BitCoins are unique and cannot be duplicated, they only exist as an electronic analog a kind of physical coin. This limits the expansion of BitCoin (Hanley 2014). For example, the absence of BitCoin denominated credit cards and consumer loans do not allow purchases on credit - a method widely used in most developed retail markets, which limits the expansion of BitCoin as a medium of exchange (Yermack 2014).

We may conclude that in the absence of BitCoin denominated credit, BitCoin growth is seriously constrained, as the share of credit transactions is rather high (and growing) in modern societies (Table 2).

\subsection{Unit of account}

To serve the function of a unit of account, as any other currency, BitCoin should be able to measure the relative value of goods and services and other transactions (e.g. debts) in the economy. We identify two key characteristics of BitCoin as a unit of account, which differ substantially from traditional currencies: divisibility and price volatility (see Table 2 for summary).

\subsubsection{Divisibility}

An important distinguishing feature of BitCoin is its nearly infinite divisibility, implying that prices may be quoted in four or more decimal places. Indeed, divisibility is a necessary characteristic of a currency to accommodate the valuation for all types and sizes of transactions. 
On the other hand, the price differences in the magnitude of several decimal places (e.g. four or more) may be confusing to consumers and may pose problems to them to comprehend and compare relative prices of goods and services. For comparison most world currencies use no more than two decimal points for price quotations (Yermack 2014).

We may conclude that, generally, due to almost infinite divisibility possibilities of BitCoin its use and popularity may grow compared to standard currencies, although small price denominations may reduce ability of consumers to accurately distinguish relative prices (Table 2).

\subsubsection{Price volatility}

BitCoin prices show extremely high short-run volatility, which diminishes its ability in representing an effective unit of account (Yermack 2014). The frequent BitCoin price changes cause direct and indirect costs to businesses and consumers. Businesses that use BitCoin have to adjust prices frequently, otherwise they may realize decrease in returns (because of underpriced goods and services) or loss of competitiveness (because of overpriced goods and services). This is particularly problematic for businesses trading outputs in BitCoins, while paying for production factors and intermediate inputs in local standard currency (e.g. US dollar, Euro, Yen, British Pound), causing discrepancy in relative prices between outputs and inputs in presence of high BitCoin price volatility. Frequent price changes in turn become confusing to consumers, as it becomes more difficult to spot the true relative prices of goods and services. ${ }^{6}$

Although, high price volatility of BitCoin may inflict risk to its holders, entrepreneurial innovations provide alternative solutions which diminish the price volatility risk. For example, market exchange pricing may facilitate price setting to businesses (retailers) and widen spending options to consumers. The market exchange pricing enable retailers to set prices in one currency (e.g. US dollar, Euro, Yen, British Pound) while displaying them to consumers simultaneously and automatically updated in more currencies, including in BitCoin, reflecting the current market exchange rates. This system makes relatively costless price tracking in BitCoin to businesses that reflect up-to-date market exchange rate particularly for online sales. Another example includes instantaneous exchange facilities which enable retailers to accept BitCoin as payment without actually receiving BitCoins. This system involves a third party which intermediates the exchange of BitCoins, paid by consumers, to standard currency, which is received by the retailer (e.g. US dollar, Euro, Yen, British Pound). Because sellers never actually receive BitCoins, they avoid the BitCoins exchange risk; the exchange risk is beard by the intermediaries which receive a fee in return (Luther and White 2014).

In the context of BitCoin use as a unit of account, the extremely high price volatility reduces BitCoin power to convey accurately the relative prices of goods

\footnotetext{
${ }^{6}$ A related problem pointed by Yermack (2014) is linked to relatively high diversity of BitCoin prices across different exchanges and web quotations at any given time. This variation in prices poses problem to establish a valid reference point for price setting for both consumers and businesses.
} 
and services in the economy (Table 2). If, as argued by Luther and White (2014), financial developments are providing alleviating options for addressing the issue of BitCoin price volatility, then the adverse impacts of price volatility may decline in future.

\subsection{Store of value}

The value of the money must remain stable over time to allow their use in exchanges in different points of time. We identify two features of BitCoin as store of value, which differ substantially from traditional currencies: non-inflationary supply and cyber security (see Table 2 for summary).

\subsubsection{Non-inflationary supply}

Standard currencies are usually inflationary, meaning that their value reduces over time and thus diminishes the ability of the currency to function as a store of value. In contrast, an important advantage of BitCoin is the protection against inflation as a safe haven from government interference. Given that under the current mechanism the future number of BitCoins is capped to a maximum amount of 21 million units with no expansion possible beyond this amount, BitCoin will be exposed to deflationary pressures if its use as an investment alternative or for use as a medium of exchange increases. ${ }^{7}$ On the one hand, this is beneficial to BitCoin holders, as it will make them richer over time. On the other hand, the expectation of higher future value of BitCoins may lead to its hoarding in anticipation of higher price which in turn may reduce its use in exchanges of goods and services. Meiklejohn et al. (2013) find that 2011 was a break point for spending-versus hording of BitCoin. After this date the majority of BitCoins (more than $60 \%$ ) that were received were spent within less than 1 month period. The hording prior to 2011 took many BitCoins out of circulation meaning that most of BitCoins from this period are in the "sink" addresses that have never been spent (Ron and Shamir 2013).

We may conclude that, in the absence of inflationary pressures, BitCoin popularity should increase compared to standard currencies, although deflationary pressure may act as a countervailing force (Table 2).

\subsubsection{Cyber security}

One of the main threats to BitCoin ability to preserve its value to the holders is the issue of cyber security. In the past, many BitCoin owners have lost their virtual money through online theft. Standard currencies give a possibility to protect it against theft either by physically hiding it (e.g. under mattresses, in a safe) or by depositing it in a bank. BitCoin is a virtual currency and thus it cannot be physically

\footnotetext{
7 Moreover, BitCoins which were accidentally lost or destroyed can never be replaced, resulting in shrinkage of the money base and leading to enhanced deflationary trend. For example, the bitomat exchange, one of the largest exchanges, lost around \$200 thousand worth of BitCoins (at the exchange rate at the time) due to human error by accidentally erasing cloud server where the wallet was stored (Barber 2012).
} 
hidden. BitCoins must be held in computer accounts known as virtual wallets. The security of BitCoin wallets has often been a major problem. Even BitCoin exchanges were subject to cyber-attacks and thefts as they are common target of hackers. The most prominent example is the collapse of the largest BitCoin exchange, MtGox, in February 2014 allegedly leading to a loss of 850 thousand BitCoins (Böhme et al. 2015; Yermack 2014). Moore and Christin (2013) find that $45 \%$ of the total studied BitCoin exchanges closed down, and $46 \%$ of the exchanges that closed did not reimburse any claims to consumers.

In the context of BitCoin use as a store of value, BitCoin sensitivity to cyberattacks and thefts reduces trust in BitCoin as a currency and hence impedes its growth and chances of becoming a global currency (Table 2).

\section{BitCoin price and its volatility}

Among all BitCoin features identified and discussed above, eventually, price volatility is the one with the largest differences compared to the major world currencies, such as US dollar, Euro, Yen, British Pound. As shown in Fig. 1, since its introduction in 2009, BitCoin price has shown extremely high volatility; it has increased from zero value at the time of its inception in 2009 to around $\$ 1100$ at the end of 2013 when it reached the peak followed by a decline to around $\$ 225$ (see Fig. 1). For comparison, the fluctuation of exchange rate between US dollar and Euro has not exceeded a $\pm 20 \%$ bound in the same 6 year period (see Fig. 1). Such market volatility with extreme price movements $( \pm 8000 \%)$ makes BitCoin of little use as a unit of account.

One consequence of such extreme price volatility is that BitCoin may not succeed as a global currency to be accepted by suppliers and consumers, as it may fail to accurately convey relative prices of goods and services in the economy and may generate uncertainties to its holders due to its inability to preserve stable value over time. Given that BitCoin is a relatively new currency, its price formation is not well understood yet. In order to better understand reasons for such extremely high price volatility, in this section we attempt to identify the drivers of BitCoin price and estimate their relative importance econometrically.

\subsection{Drivers of BitCoin price}

The existing studies in the literature (e.g. Buchholz et al. 2012; Kristoufek 2013; van Wijk 2013; Bouoiyour and Selmi 2015), suggest three types of drivers determining BitCoin price formation: (1) market forces of BitCoin supply and demand, (2) BitCoin attractiveness, and (3) global macroeconomic and financial developments.

\subsubsection{Driver 1: market forces of BitCoin supply and demand}

According to Buchholz et al. (2012), one of the key drivers of BitCoin price is the interaction between BitCoin supply and demand on the BitCoin market. According 
to the quantity theory of money, the BitCoin supply is determined by the total stock of BitCoin in circulation. The BitCoin demand is represented by the size of BitCoin economy (i.e. its use in exchanges) and the velocity of BitCoin circulation. The BitCoin velocity measures the frequency at which one unit of BitCoin is used for purchase of goods and services. The quantity theory implies that the price of BitCoin decreases with the velocity and the stock of BitCoins, but increases with the size of BitCoin economy and the general price level.

The demand for BitCoin is primarily driven by its value as a medium of exchange. BitCoin does not have intrinsic value like commodity currency such as gold standard. For example, the key difference between the gold standard and BitCoin is that the demand for BitCoin is driven solely by its value in future exchange, whereas the demand for commodity currency is driven by both its intrinsic value and its value in future exchange. The BitCoin supply is given by the total stock of units put in circulation, which is publicly known and is fixed in the long-run. Whereas BitCoin supply is exogenous, the supply of gold is endogenous, as it responds to changes in production technology (e.g. mining technology for gold) and returns. Given the exogeneity of BitCoin supply, the primary driver of its price developments is expected to be the demand side shocks. By altering expectations of future use in exchanges, such shocks to demand have the potential to produce large swings in the BitCoin price (Luther and White 2014).

\subsubsection{Driver 2: BitCoin attractiveness}

There are several BitCoin-specific factors which, in addition to traditional currency price determinants, such as market supply and demand, determine its demand. This is partially linked to the fact that BitCoin has been created relatively recently and to the nature of the currency. (Barber et al. 2012; Buchholz et al. 2012; Kristoufek 2013; van Wijk 2013; Bouoiyour and Selmi 2015).

First, BitCoin price may be affected by the risk and uncertainty of the whole BitCoin system. Given that BitCoin is a fiat currency and thus intrinsically worthless, it does not have an underlying value derived from consumption or its use in production process (such as gold). The value of a fiat currency is based on trust that it will be valuable and accepted as a medium of exchange also in future (Greco 2001; Kovenock and Vries 2002). ${ }^{8}$ The expectations about trust and acceptance are particularly relevant for BitCoin which, being a relatively new currency, is in the phase of establishing its market share by building credibility among market participants.

Second, being a virtual currency, BitCoin is more vulnerable to cyber-attacks than traditional currencies, which can destabilize the whole BitCoin system and eventually lead to a collapse of BitCoin. Such attacks have been frequently occurring in the BitCoin system in the past (Barber et al. 2012; Moore and Christin 2013). As mentioned above, Moore and Christin (2013) examined 40 BitCoin

\footnotetext{
${ }^{8}$ Given that people consider a currency valuable if they expect others to do so, for a decentralized currency, such as BitCoin, trust depends on a belief that the rules of the currency will be stable over time.
} 
exchanges and found that 18 have been closed down after cyber-attacks, while the world's largest BitCoin exchange, collapsed in 2014 due to a cyber-attack.

Third, BitCoin price may be affected by its attractiveness as an investment opportunity for potential investors. According to Gervais et al. (2001), Grullon et al. (2004) and Barber and Odean (2008), potential investors' decisions may be affected by an increase or decrease of attention in the news media. The role of information is particularly important in the presence of many alternative investment choices and positive search costs. Given that investment demand depends on the costs associated with searching for information for potential investment opportunities available on the market, such as, the stock exchange, those investment opportunities which are under a particular attention of news media may be preferred by potential investors, because they reduce search costs. In turn, increased investment demand for BitCoin may exercise upward pressure on BitCoin price. Indeed, Lee (2014) finds such evidence for BitCoin, whereby the alteration of positive and negative news generated high price cycles. This implies that the attention-driven investment behavior can affect BitCoin price either positively or negatively, depending on the type of news that dominate in the media at a given point of time.

\subsubsection{Driver 3: global macroeconomic and financial developments}

Van Wijk (2013) stresses the role of global macroeconomic and financial development, captured by variables such as stock exchange indices, exchange rates, and oil prices measures in determining BitCoin price. The impact of macroeconomic and financial indicators on BitCoin price may work through several channels. For example, stock exchange indices may reflect general macroeconomic and financial developments of the global economy. Favorable macroeconomic and financial developments may stimulate the use of BitCoin in trade and exchanges and thus strengthen its demand, which may have positive impact on BitCoin price.

Inflation and price indices are other type of indicators capturing important macroeconomic and financial developments. According to Krugman and Obstfeld (2003) and Palombizio and Morris (2012), oil price is one of the main sources of demand and cost pressures, and it provides an early indication of inflation development. Thus, when the price of oil signals potential changes in the general price level, this may lead to depreciation (or appreciation) of BitCoin price. Also the exchange rate may reflect inflation development and thus impact positively BitCoin price as indicated above.

According to Dimitrova (2005), there could be also negative relation between a currency's price and macro financial indicators. A decline in the stock prices induces foreign investors to sell the financial assets they hold. This leads to a depreciation of the underlying currency, but may stimulate BitCoin price, if investors substitute investment in stocks for investment in BitCoin. Generally, investors' return on stock exchange may capture opportunity costs of investing in BitCoin. Hence, in this case the stock exchange indices are expected to be positively related to BitCoin price. 


\subsection{Econometric approach}

To account for the three drivers identified in the previous section, we specify the following BitCoin price equation:

$$
p_{t}^{B}=\beta_{0}+\beta_{1} p_{t}+\beta_{2} y_{t}+\beta_{3} v_{t}+\beta_{4} b_{t}+\beta_{5} a_{t}+\beta_{6} m_{t}+\varepsilon_{t}
$$

where $t$ is time subscript, $p_{t}^{B}$ is BitCoin price (i.e. US dollar per unit of BitCoin), $p_{t}$ is the general price level of goods and services in the economy (e.g. exchange rate), $y_{t}$ is size of BitCoin economy, $v_{t}$ is BitCoin velocity and measures the frequency at which one unit of BitCoin is used for purchase of goods and services, $b_{t}$ represents the total stock of BitCoins in circulation, $a_{t}$ captures BitCoin attractiveness, $m_{t}$ captures macroeconomic and financial indicators, and $\varepsilon_{t}$ is an error term.

The first four variables $p_{t}, y_{t}, v_{t}$, and $b_{t}$ account for demand and supply drivers of BitCoin price (driver 1). Following the quantity theory of money we expect that coefficients $\beta_{1}$ and $\beta_{2}$ would be positive, whereas $\beta_{3}$ and $\beta_{4}$ would be negative. In addition, given that BitCoin supply is largely predefined, the total stock of BitCoins in circulation, $b_{t}$, is a semi- exogenous variable, and implying that the impact of coefficient $\beta_{4}$ on BitCoin price should be small and/or statistically not significant. Variable $a_{t}$ captures the BitCoin attractiveness (driver 2). As discussed above, the coefficient $\beta_{5}$ associated to this variable can be either negative or positive, for example, as both positive and negative news attract investors' and users' attention. Finally, variable $m_{t}$ represents global macroeconomic and financial developments (driver 3). According to the previous findings discussed above, we expect the sign of the coefficient $\beta_{6}$ to be either positive or negative depending on the type of macroeconomic variable.

The econometric model (1) contains mutually interdependent variables-BitCoin price and its explanatory variables. The estimation of non-linear interdependencies among interdependent time series in presence of mutually correlated variables is subject to an issue of endogeneity (Lütkepohl and Krätzig 2004). To circumvent the issue of endogeneity, we follow the general approach in the literature to analyze the causality between endogenous time-series and specify a multivariate Vector Auto Regressive (VAR) model (Lütkepohl and Krätzig 2004).

According to Engle and Granger (1987), regressions of interdependent and nonstationary time series may lead to spurious results. In order to avoid spurious regression, it is important to test the properties of the time series involved. Therefore, in the first step, the stationarity of time series is determined, for which we use two unit root tests: the augmented Dickey-Fuller (ADF) test and the PhillipsPerron (PP) test. The number of lags that we use for each dependent variable is determined by the Akaike Information Criterion (AIC). If two individual time series are non-stationary, their combination may be stationary (Engle and Granger 1987). In this special case, the time series are considered to be cointegrated, implying that there exists a long-run equilibrium relationship between them.

In the second step, we employ the Johansen's cointegration method to examine the long-term relationship between the price series. The number of cointegrating vectors is determined by the maximum eigenvalue test and the trace test. Both tests 
use eigenvalues to compute the associated test statistics. We follow the Pantula principle (Pantula 1989) to determine whether a time trend and a constant term should be included in the model.

In the third step, we estimate a vector error correction model for those series that are cointegrated (Johansen and Juselius's 1990). It includes an error correction term indicating the speed of adjustment of any disequilibrium towards a long-term equilibrium state. In other words, the error correction term captures the changes in the BitCoin price required to eliminate the past deviation of the prices from the equilibrium levels.

As usual, in order to ensure the adequacy of the estimated models, we implement a series of specification tests: Lagrange-multiplier (LM) test for autocorrelation in the residuals; Jarque-Bera test to check if the residuals in the Vector-Error Correction (VEC) are normally distributed and a test of stability of the model.

\subsection{Data and variable construction}

In order to construct the dependent variable, we use data for BitCoin price, $p_{t}^{B}$, denominated in US dollar (BitCoin price). We use the historical number of total BitCoins (number of BitCoins) which have been mined to account for the total stock of BitCoins in circulation, $b_{t}$. We use two alternative proxies for the size of BitCoin economy, $y_{t}$ : the total number of unique BitCoin transactions per day (number of transactions), and the number of unique BitCoin addresses used per day (number of addresses). Following Matonis (2012), we proxy the monetary velocity of BitCoin circulation, $v_{t}$, by BitCoin days destroyed for any given transaction (days destroyed). This variable is calculated by taking the number of BitCoins in transaction and multiplying it by the number of days since those coins were last spent. All these data are extracted from quandl.com. To measure the price level of global economy, $p_{t}$, we use exchange rate between the US dollar and the Euro (exchange rate) extracted from the European Central Bank. We use exchange rate between the US dollar and the Euro, because in our data BitCoin price is denominated in US dollar. For example, if the US dollar would appreciate against the Euro, most likely it will also appreciate against the BitCoin. Consequently, an increase in the exchange rate between the Euro and the US dollar would lead to a decrease in the amount of US dollar that have to be paid for one BitCoin, which decreases its price.

In order to capture BitCoin attractiveness, $a_{t}$, we follow Kristoufek (2013) and use the volume of daily BitCoin views on Wikipedia (views on Wikipedia). According to Kristoufek (2013), the frequency of searches related to the virtual currency is a good measure of potential investors' interest in the currency. However, the online search queries, such as Wikipedia views, may measure both investors' and users' interest in BitCoin, as it captures information's demand about the currency but it does not differentiate on whether the information is used to guide investment decisions or online BitCoin denominated exchange of goods and services. In addition, we also construct a variable capturing the number of new members (new members) and new posts on online BitCoin forums (new posts) extracted from bitcointalk.org. As explained above, the variable new members 
captures the size of the BitCoin economy but also attention-driven investment behavior of new BitCoin members. The variable new posts captures the effect of trust and/or uncertainty, as it represents the intensity of discussions among members.

To account for global macroeconomic and financial developments, $m_{t}$, we follow van Wijk (2013) and use oil price (oil price) and the Dow Jones stock market index (Dow Jones). ${ }^{9}$ Oil prices are extracted from the US Energy Information Administration, and Dow Jones index is extracted from the Federal Research Bank of St. Louis.

\subsection{Estimation results}

Following the drivers of BitCoin price formation identified in Sect. 3.1, we estimate four sets of econometric models. The differences in the specifications between the estimated models are summarized in Table 3. Models 1.1-1.5 capture BitCoin supply-demand interactions and their impact on BitCoin price. Model 2.1 estimates the impact of BitCoin attractiveness indicators. Model 3.1 estimates the impact of global macroeconomic and financial developments. Models 4.1-4.9 interact the three types of BitCoin price drivers.

The estimation results are reported in Tables 4, 5, 6 and 7. Tables 4 and 5 report the short-run impact of different determinants on BitCoin price. The short-run effects represent short-run dynamics of variables in the cointegrated system. They describe how the time series react when the long-run equilibrium is distorted. According to the results reported in Tables 4 and 5, a number of variables have statistically significant short-run effect on BitCoin price adjustments. In particular, this is the case for own price effects, the stock of total BitCoins (number of BitCoins) BitCoin days destroyed (days destroyed) and Wikipedia views (views on Wikipedia).

Tables 6 and 7 show the long-run impacts of different determinants on BitCoin price. According to the results reported in Tables 5 and 6, the long-run relationship between BitCoin price and the explanatory variables considered in the estimated models is stronger than the short-run impact. In the following we discuss the longrun results with respect to the three drivers of BitCoin price formation.

\subsubsection{Results for driver 1: market forces of BitCoin supply and demand}

The first major observation arising from the estimates reported in Tables 6 and 7 is that the market forces of supply and demand have an impact on BitCoin price. Generally, the demand side variables (e.g. days destroyed, number of addresses) appear to exert a more pronounced impact on BitCoin price than the supply side drivers (e.g. number of BitCoins). According to the results reported in Table 6, an increase in the stock of BitCoins (number of BitCoins) leads to a decrease in BitCoin price (model 1.2), whereas an increase in the size of the BitCoin economy (number

\footnotetext{
9 The Dow Jones Index is an industrial average that captures 30 major corporations on either the NYSE or the NASDAQ.
} 


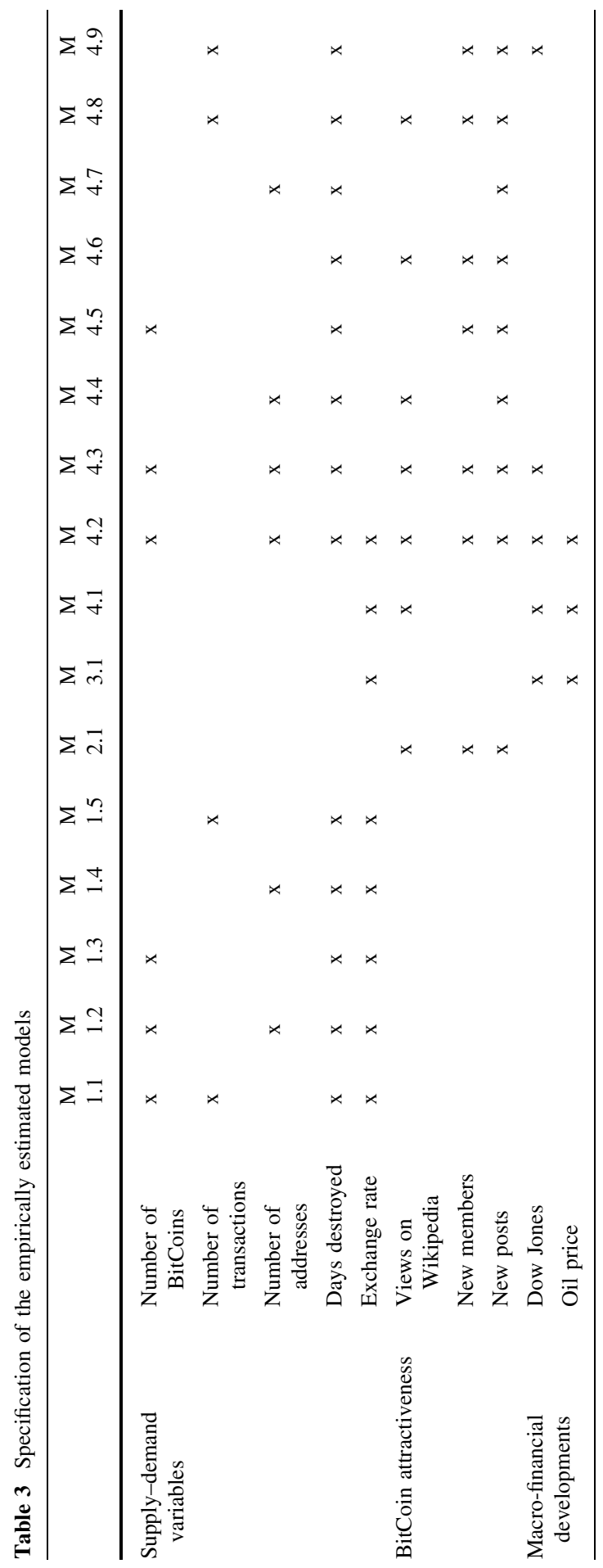




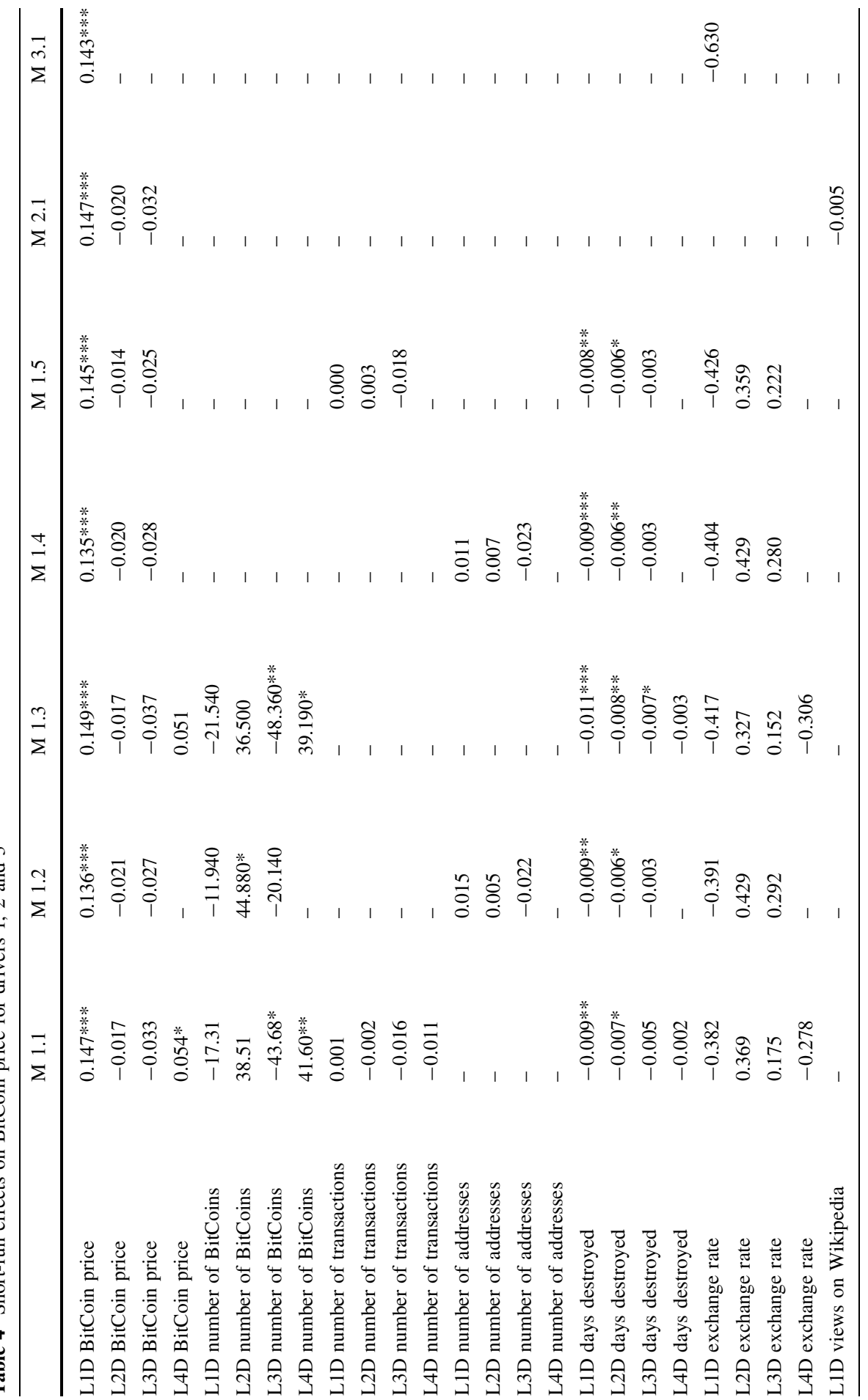




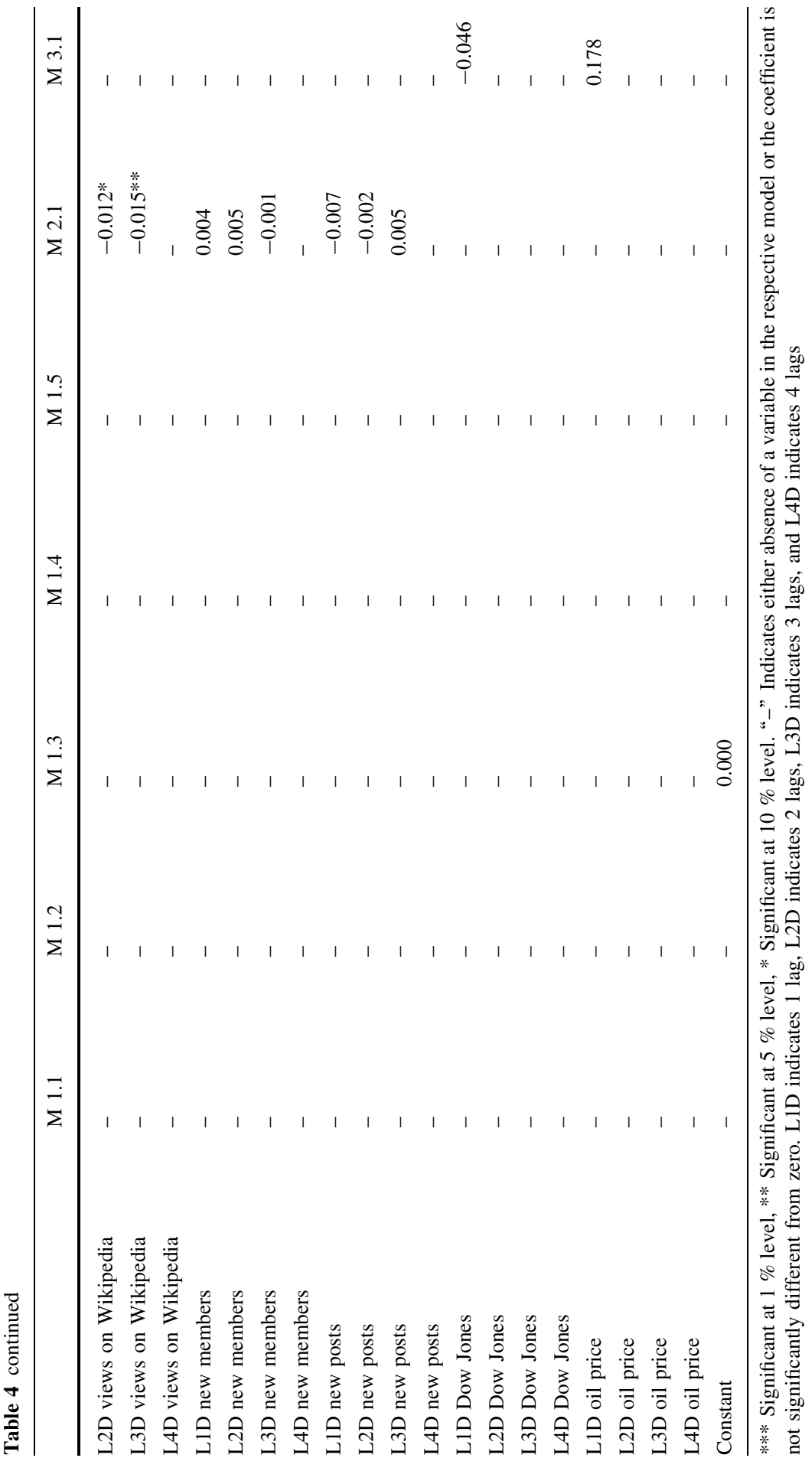




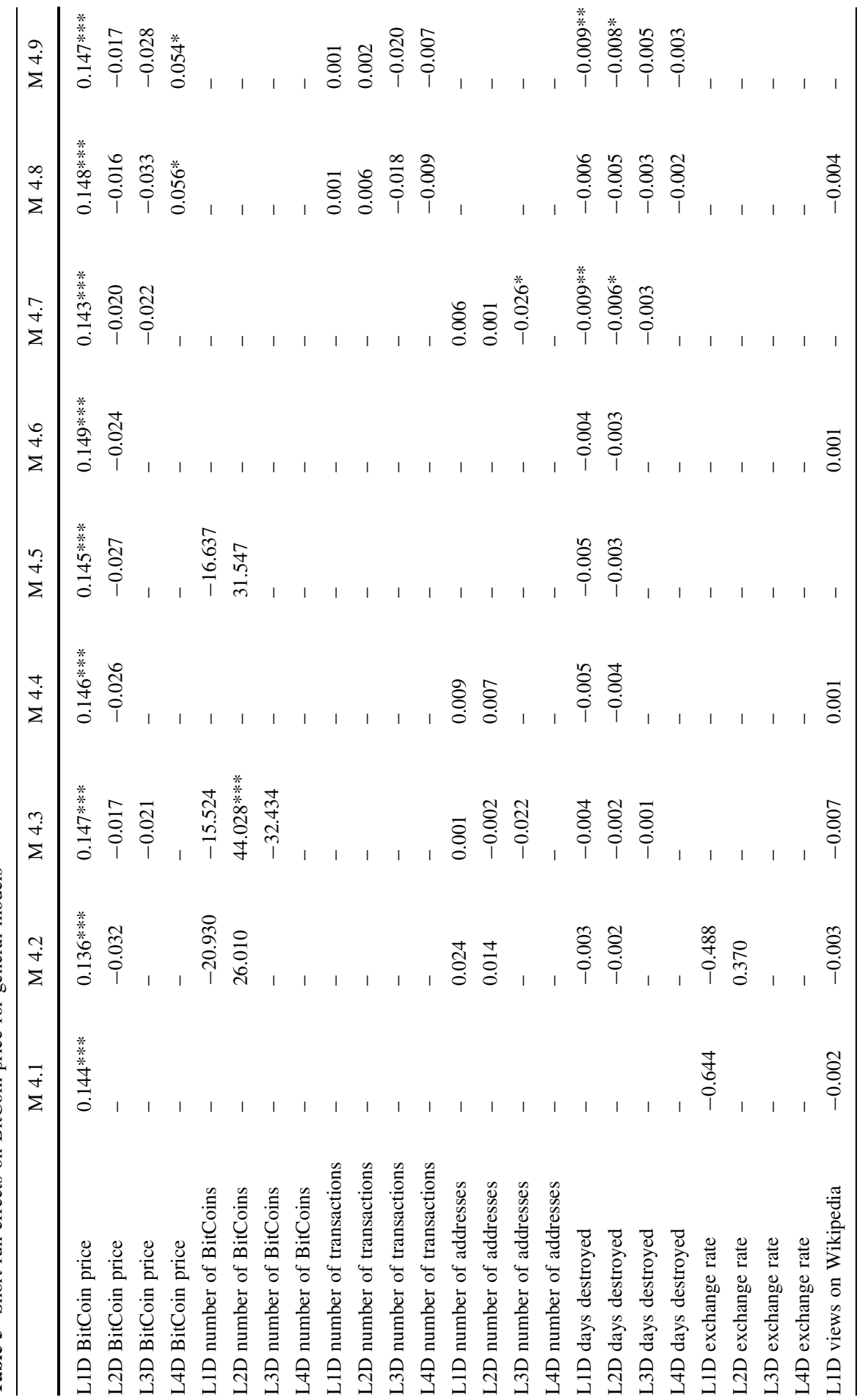




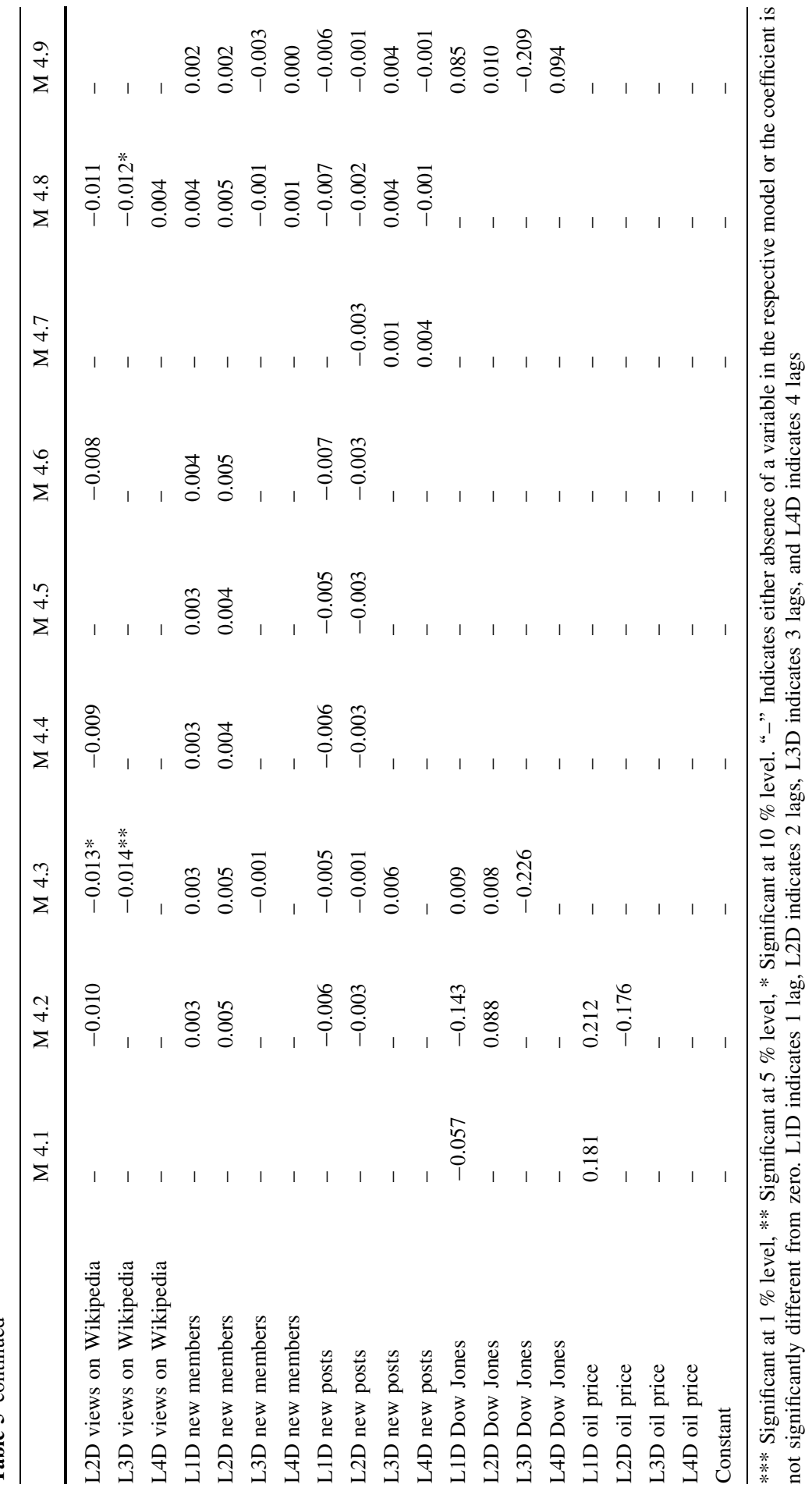




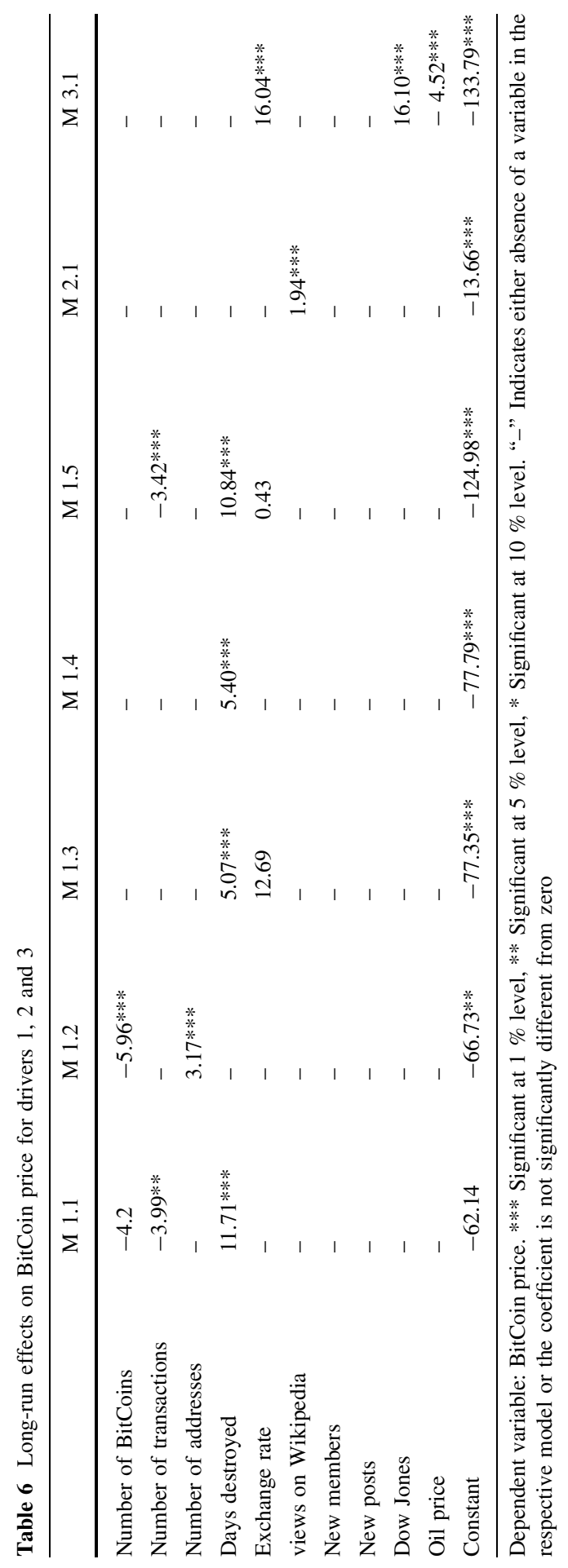




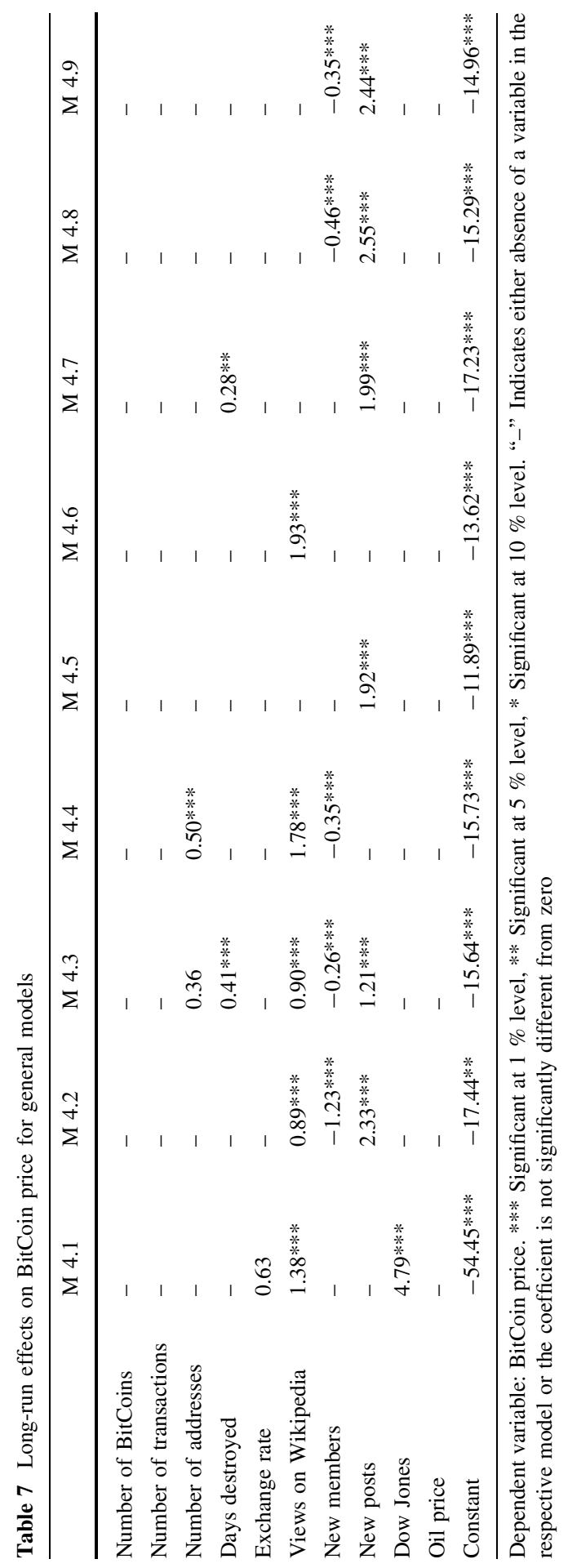


of addresses) and its velocity (days destroyed) lead to an increase in BitCoin price (models 1.1, 1.2, 1.3, 1.4, 1.5, 4.3, 4.4, 4.7). These results are in line with our expectations. Contrary to our expectations, the alternative variable that captures the size of the BitCoin economy (number of transactions) has negative impact on BitCoin price in models 1.1 and 1.5. However, this variable is not significant in the more general models (models 4.1-4.9), implying that its impact on BitCoin price is negligible.

Although, the sign of the estimated coefficients for market forces of BitCoin supply and demand is in line with our hypothesis (except for the variable number of transactions in models 1.1 and 1.5), the statistical significance and magnitude of the estimated coefficients decreases in most models, when accounting for the impact of BitCoin attractiveness and global macroeconomic and financial developments (models 4.1-4.9 in Table 7). The supply-demand variables are statistically significant in models $4.3,4.4$ and 4.7 , but have a considerably lower magnitude of the estimated impact than in models 1.1-1.5, which capture only market forces of supply and demand. This could be explained by the fact that part of the BitCoin price variation explained by the supply-demand variables is absorbed by other variables in more general specifications (models 4.1-4.9).

\subsubsection{Results for driver 2: BitCoin attractiveness}

The strongest and statistically the most significant impact on BitCoin price is estimated for variables capturing the impact of BitCoin attractiveness: views on Wikipedia, new members and new posts (models 2.1 and models 4.1-4.9). Variable new members has negative impact on BitCoin price, implying that attention-driven investment behavior of new investors dominates. Variable new posts has positive impact on BitCoin price, reflecting an increasing acceptance and trust in BitCoin captured by the intensity of discussion between BitCoin users. This may reflect declining transaction costs and uncertainty for investors, which increases investment demand for BitCoin and hence its price.

Consistent with the findings of Kristoufek (2013), Wikipedia views have a statistically significant impact on BitCoin price. This variable is significant and has positive impact in all models (except for model 4.8). These results are in line with our expectations and with the remarkable Wikipedia article traffic statistics, according to which on average BitCoin article on Wikipedia is being viewed 200,000 times per month, and is ranked among the top 100 most viewed articles. Only 88 English-language Wikipedia articles have more traffic than the BitCoin article, out of a total of approximately 5 million English-language articles. However, the interpretation of Wikipedia views is not straightforward, as it may capture various effects. On the one hand, Wikipedia views may represent attention devoted by potential users' and/or investors' to BitCoin likely induced by their reaction to media reporting on BitCoin. The attention effect may impact either positively or negatively the BitCoin price depending on the type of news. The positive estimated coefficient associated with Wikipedia views variable implies that the impact of positive news dominates. One of the most common 
negative news reported in media about BitCoin is related the security breach (cyber-attacks) against the currency. The positive estimated results indicate that the implication of security problem appears to be offset by the positive news effect.

On the other hand, Wikipedia views may measure investors' or users' interest in BitCoin, as it captures information's demand about the currency. It may reflect changes in the knowledge about BitCoin between potential investors and/or users, thus leading to a higher acceptance and demand for it either as an investment opportunity or as a medium of exchange. Important is that the type of individuals searching information about BitCoin on Wikipedia likely are new BitCoin users/ investors, because Wikipedia contains rather general information about BitCoin, which is known by incumbent investors or advanced BitCoin users. Both investors and users may search for information about BitCoin and thus the Wikipedia effect may capture either the users' demand for information with the aim to use it as a medium of exchange or may reflect investors' information search who perceive BitCoin as an investment opportunity (i.e. for hoarding). Kristoufek (2013) argues that the second-the hording-effect dominates because, according to him, the market forces of BitCoin supply and demand allowing for setting a "fair" price are missing, rather its price is driven by the investors' faith in the future growth and is dominated by short-term investors, trend chasers, noise traders and speculators. This argument is also in line with the sceptics on BitCoin (e.g. Velde 2013; Hanley 2014; Yermack 2014) who argue that hoarding is one of the key weaknesses of BitCoin as a currency (alongside the security problem) compared to standard currencies.

\subsubsection{Results for driver 3: global macroeconomic and financial developments}

Our findings suggest that, in contrast to previous studies (i.e. van Wijk 2013), global macro-financial developments such as the Dow Jones Index, exchange rate and oil price do not significantly affect BitCoin price in the long-run. Only in Model 3.1 the macro and financial variables (Dow Jones, oil price and exchange rate) are statistically significant (Table 6). Our results suggest that an increase in oil price leads to a decrease in the budget of consumers (and companies), implying that less money will be spent on other goods, including BitCoin. Consequently, this would lead to a decrease in demand for BitCoin, decreasing its price. These results are in line with the estimates of van Wijk (2013), who also finds statistically significant impact of macro-financial variables on BitCoin price. However, van Wijk (2013) does not account for market forces of supply and demand or BitCoin attractiveness indicators. When these factors are taken into consideration (models 4.1-4.9), their impact decreases considerably in all estimated models (except for model 4.1) (Table 7). These finding support the argument of Yermack (2014) that BitCoin is relatively ineffective as a tool for risk management against adverse market developments as its price is not responsive to macroeconomic variables meaning that it cannot be easily hedged against other assets that are driven by macroeconomic developments. 


\subsection{Comparison with previous studies}

The verification of our results through comparison with previous studies is a challenging task, as (1) the currency is relatively new and there are not many studies in the literature, which analyze BitCoin price formation; and (2) there are important differences between the used empirical proxies for variable construction and model specification across different studies. Despite these difficulties, in this section we attempt to compare our results with those reported in the literature. Generally, the study of Bouoiyour and Selmi (2015) seems to be the most comparable study to ours in terms of the employed estimation techniques, underlying data and explanatory variables. Similarly to our paper, they control for all three sets of drivers: market forces, attractiveness indicators and macro variables. Other studies are less comparable to our approach mainly due to differences in model specification, as most of them study the impact of each BitCoin price driver separately; they do not consider interactions between them. For example, Kristoufek (2013) considers only BitCoin attractiveness indicators, whereas Van Wijk (2013) includes only the macroeconomic drivers. Buchholz et al. (2012) account for the impacts of BitCoin supply and demand, and BitCoin attractiveness, but does not account for global macroeconomic and financial developments.

The impacts of BitCoin supply and demand (driver 1) on BitCoin price are measured by the ratio of exchange and trade transactions and velocity as (the frequency at which one unit of BitCoin is used to purchase goods) in Bouoiyour and Selmi (2015). Buchholz et al. (2012) measure BitCoin demand by price volatility. According to Bouoiyour and Selmi (2015), the estimated short-run impact of transactions on BitCoin price is positive and statistically significant $(0.129$, Table 7 in Bouoiyour and Selmi). Compared to our not significantly different from zero estimates ( -0.018 to 0.003 , models 1.1 and 1.5 in Table 4$)$ they are also smaller by around one order of magnitude. These differences may be caused by the fact that Bouoiyour and Selmi (2015) proxy for transactions using the ratio of exchange and trade transactions, whereas our proxy for transaction variable is directly constructed from the number of BitCoin transactions. Note also that our short-run estimates are not statistically different from zero. The estimated long-run impact of transactions is also positive and statistically significant at the $90 \%$ level in Bouoiyour and Selmi (2015) (0.018, Table 7 in Bouoiyour and Selmi). Surprisingly, the long-run impact of transactions is considerably lower (by one order of magnitude) than the short-run impact. In contrast, our estimates suggest that the long-run effect of transactions is negative and considerably higher (by three orders of magnitude) ( -3.99 and -3.42 , models 1.1 and 1.5 in Table 6) than the short-run effect. Note that our estimates are significant at the 95 and $99 \%$ levels, respectively). Given that our specification is more general, we use longer time series and our results are more significant, a negative long-run relationship between the number of transactions and BitCoin price is more likely.

According to Bouoiyour and Selmi (2015), the estimated short-run impact of BitCoin velocity is positive and considerably higher (2.741, Table 7 in Bouoiyour and Selmi) compared to our negative and rather small estimates ( -0.011 to -0.002 , models 1.1-1.4 in Table 4). Note, however, that the coefficient of Bouoiyour and 
Selmi (2015) is not statistically significant, whereas it is statistically significant for all estimated models with one and two lags in our results. Again, these differences may be explained by differences in the velocity variable construction and model specifications. Whereas Bouoiyour and Selmi (2015) calculate velocity as the frequency at which one unit of BitCoin is used to purchase goods, we measure velocity by the number of days needed until a BitCoin is destroyed. The estimated long-run impact of transactions is positive in Bouoiyour and Selmi (2015) as well (0.004, Table 7 in Bouoiyour and Selmi). However, it is not statistically significant. As in the case of transactions, the long-run impact of velocity is considerably lower (by three orders of magnitude) than the short-run impact in Bouoiyour and Selmi. In contrast, our estimates suggest that the long-run effect of velocity is positive and considerably higher (by two orders of magnitude) (5.07-11.71, models 1.1, 1.3, 1.4 and 1.5 in Table 6) than the short-run effect. Note that the magnitude of the estimated long-run coefficients is very stable across all for models, and they all are significant at the $99 \%$ level. Given that our specification is more general, we use longer time series and our results are more significant, a positive long-run relationship between velocity and BitCoin price is more likely.

Buchholz et al. (2012) estimate the relationship between BitCoin price and transactions, though they are interested in the reverse relationship, as they attempt to answer the question how BitCoin price shocks affect BitCoin transactions. Hence, their results are not directly comparable to our estimates. Buchholz et al. (2012) also estimate the relationship between BitCoin demand and BitCoin price, whereas BitCoin demand is proxied by price volatility. They find that an increasing price volatility leads to higher BitCoin price. The coefficient from the GARCH-in-mean model is statistically significant. Moreover, the results are different between the two analyzed periods (August 2010-June 2011 vs. June 2011-March 2012): in the first period the volatility led to a demand for BitCoins, and after the bubble burst in June 2012 the novelty of BitCoin decreased, only market participants who were averse to volatility stayed in the market, leading to no effect of volatility on BitCoin price in the second period. However, as above, their proxy for market demand-price volatility-is very different from our proxy-number of transactions-and hence cannot be directly compared with our estimates. Moreover, Buchholz et al. (2012) do not consider all types of BitCoin price drivers and interactions between them, and they use weekly data for a rather short period (82 weeks), whereas our analysis is based on daily data for a 5.5 year period, capturing the extreme price changes in 2013 and 2014 (see Fig. 1).

The impact of BitCoin attractiveness (driver 2) on BitCoin price is measured by BitCoin searches in Google in Bouoiyour and Selmi (2015) and Buchholz et al. (2012), whereas Kristoufek (2013) uses both BitCoin searches in Google and Wikipedia views.

The estimated short-run impact of searches in Google is positive and sizeable in Bouoiyour and Selmi (2015), when compared to our negative and considerably smaller estimates. These differences could be explained by differences in variable construction and model specifications. While Bouoiyour and Selmi (2015) use the number of BitCoin searches in Google to proxy for investors' attractiveness, we use the number of views of BitCoin site on Wikipedia. The estimated long-run impact of 
searches in Google is positive and smaller than the short-run estimates $(0.143$ and 0.527, respectively, Table 7 in Bouoiyour and Selmi) but statistically not significantly different from zero. Again, our long-run estimates (1.94, model 2.1 in Table 6) are of higher order of magnitude with respect to our short-run estimates. Note that our model specification is more general, and our estimates are significant at the $99 \%$ level, which suggests that they may be more precise.

Kristoufek (2013) studies the impact of BitCoin attractiveness on its price by using BitCoin searches in Google and Wikipedia views. He finds that the increased interest in BitCoin measured by BitCoin searches in Google increases its price. As the interest in BitCoin increases, the demand increases as well causing the prices to increase. The estimated positive impact is in line with our results. The relationship between Wikipedia views and BitCoin turns out to be statistically not significant in Kristoufek (2013). In contrast, our long-run results suggest a positive and statistically significant impact of Wikipedia views on BitCoin price (1.94, model 2.1 in Table 6). The statistically significant results of Kristoufek (2013) may be caused by the fact that his analysis is based on a rather short period (2 years) compared to a 5.5 year period in our study as well as they do not control for other BitCoin price drivers, such as BitCoin supply and demand and macroeconomic developments. Note that Kristoufek (2013) finds that the relationship is positive and statistically significant in the opposite direction. The estimated coefficient is 0.05 , which implies that a $10 \%$ change in BitCoin price is connected to a $0.5 \%$ permanent shift in the Wikipedia views.

Also Buchholz et al. (2012) use BitCoin searches in Google to estimate the impact on BitCoin. However, they estimate the relationship between Google hits and the number of BitCoin transactions, not BitCoin price. Hence, their results are not directly comparable to our estimates.

The impact of global macroeconomic and financial developments (driver 3) on BitCoin price is measured by output volume, gold price and Chinese market index in Bouoiyour and Selmi (2015). van Wijk (2013) measures global macroeconomic and financial developments by the Dow Jones index, the value of the FTSE 100, the value of the Nikkei 225, the Euro-US dollar exchange rate, the Yen-US dollar exchange rate, and several oil price indices.

According to Bouoiyour and Selmi (2015), output volume and Chinese market index have positive and statistically significant impact on BitCoin price (0.079 and 0.380, respectively, Table 7 in Bouoiyour and Selmi), whereas the impact of gold price is negative and statistically insignificant $(-0.241$, Table 7 in Bouoiyour and Selmi). Our estimates are different, as they rely on different proxies capturing global macroeconomic and financial developments: Dow Jones index and oil price $(-0.046$ and 0.178 , model 3.1 in Table 4). However, our estimates are not significantly different from zero. Both the sign and the magnitude of the estimated long-run coefficients on the output volume and Chinese market index are rather similar to short-run estimates (0.007 and 0.216, Table 7 in Bouoiyour and Selmi). The longrun effect of the gold price coefficient decreases by two orders of magnitude $(-0.002)$ compared to the short-run impact in Bouoiyour and Selmi. However, none of the long-run global macroeconomic and financial development coefficients is statistically significant in Bouoiyour and Selmi (2015). In contrast, both our global 
macroeconomic and financial development coefficients have a statistically significant impact on BitCoin price in the long-run. Whereas the impact of the Dow Jones index is positive (16.10, model 3.1 in Table 6), the impact of oil price is negative (-4.52, model 3.1 in Table 6). Note that signs of our long-run estimates are the same as those of Bouoiyour and Selmi (2015): Dow Jones index and Chinese market index have positive impact, whereas gold price and oil price have negative impact on BitCoin price. Given that our long-run estimates are statistically significant but those of Bouoiyour and Selmi (2015) are not, our estimates can be considered as more reliable.

van Wijk (2013) finds that the value of the Dow Jones has positive and statistically significant effect on BitCoin price in the long-run (0.525, Table 3.3 in van Wijk). The positive and statistically significant coefficient is in line with our results for models 3.1 and 4.1. The differences in the estimated elasticities may be caused by the fact that the analysis of van Wijk (2013) (1) does not consider other determinants of BitCoin price, such as BitCoin demand and supply, and BitCoin attractiveness, and (2) is based on a $<3$ year period, whereas our analysis is based on a 5.5 year period, capturing extreme price changes in 2013 and 2014 (see Fig. 1). The estimated impact of the Euro-US dollar exchange rate is negative and statistically significant $(-0.335$, Table 3.3 in van Wijk). The results of van Wijk (2013) suggest that, if the US dollar appreciates against Euro, it is most likely to be the case that it also appreciates against the BitCoin. Consequently, an increase in the Euro-US dollar exchange rate leads to a decrease in the amount of US dollar that have to be paid for one BitCoin, which decreases its value. In contrast, our estimates suggest a positive relationship between the Euro-US dollar exchange rate and BitCoin price. However, none of our estimated models is statistically significant (1.3, 1.5 and 4.1). Given that the estimates of van Wijk (2013) are statistically significant and plausible from an economic theory point of view, likely they are more reliable. van Wijk (2013) also estimates the impact of oil price on BitCoin price. The estimated impact is negative and statistically significant $(-0.242$, Table 3.3 in van Wijk). These results are in line with our estimates $(-4.52$, model 3.1 in Table 6). As explained above, differences between the estimates may be caused by the fact that the times series of van Wijk (2013) are only half as long as ours, not capturing the extreme price changes in 2013 and 2014, as well as they do not control for other important BitCoin price drivers.

\section{Conclusions}

Currency, or money in general, is typically defined as having three main functions: a medium of exchange, a unit of account, and a store of value. The present paper attempts to analyze BitCoin features, which may facilitate to become a global currency, as well as characteristics, which may impede the use of BitCoin as a medium of exchange, a unit of account, and a store of value.

The first function of any currency is to intermediate the exchange of goods and services. Being not a legal tender, BitCoin is fully dependent on voluntary adoption by market participants as a medium of exchange. BitCoin primary advantage 
relative to standard currencies for its use in exchanges is lower costs of transfers as there are minimal costs linked to third-party intermediaries. Although, BitCoin has shown a phenomenal growth during the last years, it still has a negligible market presence globally as a medium of exchange. Moreover, evidence tends to support the view that many of BitCoin transactions involve transfers between speculative investors and are not used in exchanges of goods and services. BitCoin faces also several other obstacles in becoming a widely used medium of exchange such as users' difficulty of procuring new BitCoins, the problem of irreversibility of BitCoin transactions, which does not allow to handle disputes, inability to grant loans denominated in BitCoin, and a relatively high level of computer knowledge required for using and understanding BitCoin.

The second function of a currency is to serve the function of a unit of account by being able to convey the relative value of goods and services in the economy. The major concern of BitCoin is its high price volatility which may reduce its power to fulfill this function accurately. However, entrepreneurial innovations such as market exchange pricing and instantaneous exchange facilities may remedy this problem but not fully eliminate it. Further, nearly-infinite sub-divisibility is a great advantage of BitCoin relative to standard currencies, though in some instances it may pose confusion among consumers given that the price quotation differentiated in the magnitude of several decimal places may be undistinguishable for them.

The third function of a currency is to serve as a store of value over time. Standard currencies are usually inflationary, while BitCoin is associated with deflationary pressures, if it becomes widely used. While this is beneficial to BitCoin holders, the expectation of higher future value of BitCoin may lead to its hoarding, which may reduce its use in exchanges of goods and services. One of the main threats to BitCoin ability to preserve the value to its holders is the security problem linked to cyber-attacks given that BitCoin is a virtual currency, its system is fully internet based and has no oversight institution entrusted to protect the system.

The identified BitCoin features are compared with traditional currencies and their possible impact on BitCoin functions as a currency. We identify several features of BitCoin as a medium of exchange, which differ substantially from traditional currencies: legal tender, fixed costs, network externalities, transaction costs, dispute resolution, credit market, anonymity and transparency. We also find two key characteristics of BitCoin as a unit of account, which differ substantially from traditional currencies: price volatility and divisibility. Finally, we identify two features of BitCoin as store of value, which differ substantially from traditional currencies: non-inflationary supply and cyber security.

Among all BitCoin features identified and analyzed in this paper, eventually, price volatility is the one with the largest differences compared to standard currencies, such as US dollar, Euro, Yen, British Pound. As shown in Fig. 1, since its introduction in 2009, BitCoin price has shown extremely high volatility. One consequence of such extreme price volatility is a threat for BitCoin being accepted as a global currency, as it may fail to accurately convey relative prices of goods and services in the economy and may generate uncertainties to its holders due to its inability to preserve stable value over time. Indeed, a desirable property of any monetary mean is that it holds its value over short-to-medium periods of time, in 
order not to create distortion when used as a medium of exchange in transactions, a unit of account, and a store of value. Large price movements alter the purchasing power causing risk and costs to firms and consumers. Given that BitCoin is a relatively new currency, its price formation is not well understood yet, as there are only few studies on BitCoin price formation available in the literature.

In order to better understand reasons for such extremely high price volatility, in the second part of the paper we attempt to identify drivers of BitCoin price and estimate their importance econometrically. The previous literature suggests three types of drivers determining BitCoin price development: (1) market forces of BitCoin supply and demand, (2) BitCoin attractiveness, and (3) global macroeconomic and financial developments. We apply time-series analytical mechanisms to these drivers using daily data for the 2009-2014 period.

Our empirical results confirm that market forces of BitCoin supply and demand have an impact on BitCoin price, implying that, to a certain extent, the formation of BitCoin price can be explained in a standard economic model of currency price formation. In particular, the demand-side drivers, such as the size of the BitCoin economy and the velocity of BitCoin circulation, were found to impact BitCoin price. Given that BitCoin supply is exogenous, likely, the development of the demand side drivers will be among the key determinants of BitCoin price also in the future potentially lading to deflationary pressures if BitCoin use expands.

However, the impact of demand-side drivers on the BitCoin price somehow reduces when controlling for BitCoin attractiveness, which implies that these drivers appear to be relatively more important. In fact, we cannot reject the hypothesis extensively emphasized in the literature that investor speculations are also affecting BitCoin price. The statistically significant impact of Wikipedia views on BitCoin price could be an indicator of speculative short-run behavior of investors, or it may capture the expansion of the demand as a medium of exchange of the BitCoin. Additionally, we find that also new information impact BitCoin price positively, which may be a result of an increased trust among users. As such, speculative trading of BitCoins is not necessarily an undesirable activity, as it may generate benefits in terms of absorbing excess risk from risk averse participants and providing liquidity on the BitCoin market. A negative side of the short-run speculative investment is that it may increase price volatility and create price bubbles which has adverse implication for BitCoin users. A further negative side of the long-run speculative investment is more extensive hoarding of BitCoins which may reduce its use in exchanges. The success of BitCoin thus also hitches on its ability to reduce the potential negative implications of such speculations and expand the use of BitCoin in trade and commerce.

Finally, our econometric estimates do not support previous findings that the global macro-financial development may be driving BitCoin price. In fact, once we control for supply-demand variables and BitCoin's attractiveness for investors linked to BitCoin, the impact of global macro-financial development captured by the Dow Jones Index, exchange rate and oil price becomes statistically insignificant. Because macroeconomic changes are not reflected in BitCoin price movements its price volatility cannot be easily hedged. This is in contrast to standard currencies 
which are heavily driven by macro developments and hedging option is available and widely used.

In summary, our study has shown that there is a disagreement in the literature on whether BitCoin can become a global currency. Overall, negative assessments about BitCoin as a currency tend to prevail given that several BitCoin characteristics identified in the paper impede its use as a currency and thus constrain its expansion globally. However, as outlined in the paper some BitCoin characteristics give its predisposition to be adopted at least in some segment of money market if not in a wider context. In particular, BitCoin may have a high relative comparative advantage with respect to standard currencies in countries with unstable financial system (e.g. in developing countries), and may provide an alternative to standard currencies in countries with poor and not widely available financial services, nonconvertible currency, expensive financial services and high administrative burden in opening an account. In addition, BitCoin may represent a cost-effective remittance system in developing countries, were traditional transfers are very expensive and the banking system is underdeveloped and unsecure. Given that BitCoin transfers can be done with relatively minimal cost and resource requirements and are independent of geographical location or banking system in place, they are ideally positioned to serve as an efficient international remittance system. ${ }^{10}$

The BitCoin technology-BlockChain ${ }^{11}$-opens up several other possibilities and technological innovations, including micropayments, crowdfunding, distributed exchanges, smart property, property registry, ticketing and secure voting systems. For example, BlockChain technology could provide a way to track the unique history of individual devices, by recording a ledger of data exchanges between it and other devices, web services, and human users. Similarly, BlockChain could enable smart devices to become independent agents, autonomously conducting a variety of transactions. For example, a vending machine could not only monitor and report its own stock, but also solicit bids from distributors and pay for the delivery of new items automatically-based on the purchase history of its customers. Further, the disruptive innovation of BitCoin provides the potential to give citizens direct control over their financial activities by removing costly — and sometimes obscure-intermediation layers fostering financial inclusion .

Acknowledgments The authors are grateful to Tony Tam for providing access to the BitCoin data of Bitcoinpulse. We gratefully acknowledge financial support from the research project VEGA1/0797/16. The authors are solely responsible for the content of the paper. The views expressed are purely those of the authors and may not in any circumstances be regarded as stating an official position of the European Commission.

\footnotetext{
${ }^{10}$ An example of the BitCoin based system for remittance transfers is BitPesa. BitPesa is an online payment platform that uses BitCoin to offer money transfers to and from East Africa (Folkinshteyn et al. 2015).

11 BlockChain is a public ledger of all BitCoin transactions that have ever been executed; it is constantly growing as 'completed' blocks are added to it with a new set of recordings.
} 
Open Access This article is distributed under the terms of the Creative Commons Attribution 4.0 International License (http://creativecommons.org/licenses/by/4.0/), which permits unrestricted use, distribution, and reproduction in any medium, provided you give appropriate credit to the original author(s) and the source, provide a link to the Creative Commons license, and indicate if changes were made.

\section{References}

Barber BM, Odean T (2008) All that glitters: the effect of attention and news on the buying behavior of individual and institutional investors. Rev Financ Stud 21(2):785-818

Barber S, Boyen X, Shi E, Uzun E (2012) Bitter to better-how to make BitCoin a better currency. In: Keromytis AD (ed) Financial cryptography and data security. Vol. 7397 of Lecture notes in computer science. Springer, Berlin. pp 399-414

Berentsten A (1998) Monetary policy implications of digital money. KYKLOS 89:89-117

Bitcoin (2015) Protect your privacy. https://bitcoin.org/en/protect-your-privacy. Accessed 27 June 2015

Bitcoin W (2015) Category: gambling. https:/en.bitcoin.it/wiki/Category:Gambling. Accessed 7 June 2015

Bitcoinhelp (2014) Using Bitcoin anonymously. Bitcoinhelp.net 21 August 2014. https://bitcoinhelp.net/ know/more/using-bitcoin-anonymously

BlockChain (2015) Number of transactions per day. BlockChain. https://blockchain.info/charts/ntransactions. July 2015

Böhme R, Christin N, Edelman B, Moore T (2015) Bitcoin: economics, technology, and governance. J Econ Perspect 29(2):213-238

Bouoiyour J, Selmi R (2015) What does Bitcoin look like? Ann Econ Finance 16, (Forthcoming)

Brito J, Castillo A (2013) Bitcoin: a primer for policymakers. Mercatus Center, George Mason University, Arlington

Bryans D (2014) Bitcoin and money laundering: mining for an effective solution. Indiana Law J 89:440-472

Buchholz M, Delaney J, Warren J, Parker J (2012) Bits and bets, information, price volatility, and demand for BitCoin. Economics 312. http://www.bitcointrading.com/pdf/bitsandbets.pdf

CoinDesk (2015a). What can you buy with BitCoins? CoinDesk 17 February 2015. http://www.coindesk. com/information/what-can-you-buy-with-bitcoins/

CoinDesk (2015b) What is Bitcoin? CoinDesk 20 March 2015. http://www.coindesk.com/information/ what-is-bitcoin/

CoinDesk (2015c) How can i buy Bitcoins? CoinDesk 20 March 2015. http://www.coindesk.com/ information/what-is-bitcoin/

Crawford D (2014) Four new ways to make Bitcoin payments anonymous. Bestvpn.com 7 May 2014. https:/www.bestvpn.com/blog/9698/four-new-ways-to-make-bitcoin-payments-anonymous/

Cuthbertson A (2015). Bitcoin now accepted by 100,000 merchants worldwide. International Business Times, February 4, 2015. http://www.ibtimes.co.uk/bitcoin-now-accepted-by-100000-merchantsworldwide-1486613

Dimitrova D (2005) The relationship between exchange rates and stock prices: studied in a multivariate model. Issues Political Econ 14:1-25

EBA (2014) EBA opinion on 'virtual currencies'. EBA/Op/2014/08, European Banking Authority

ECB (2012) Virtual currency schemes. European Central Bank, Frankfurt am Main Germany, Frankfurt

Engle RF, Granger CWJ (1987) Co-integration and error correction: representation, estimation and testing. Econometrica 55(2):251-276

EPRS (2014) Bitcoin: market, economics and regulation. Briefing 11/04/20014, European Parliamentary Research Service

FATF (2014) Virtual currencies key definitions and potential AML/CFT risks. Financial Action Task Force, FATF/OECD Paris, France, Paris

Folkinshteyn D, Lennon M, Reilly T (2015) The Bitcoin mirage: an oasis of financial remittance. J Strateg Int Stud 10:118-124

Gervais Simon, Kaniel Ron, Mingelgrin Dan H (2001) The high-volume return premium. J Finance 56:877-919

Gowrisankaran G, Stavins J (1999) Network externalities and technology adoption: lessons from electronic payments. Working papers 99-5, Federal Reserve Bank of Boston 
Greco TH (2001) Money: understanding and creating alternatives to legal tender. Chelsea Green Publishing, White River Junction, Vermont

Grinberg R (2011) BitCoin: an innovative alternative digital currency. Hastings Sci Technol Law J 4:159-208

Grullon G, Kanatas G, Weston JP (2004) Advertising, breadth of ownership, and liquidity. Rev Financial Stud 17:439-461

Hanley BP (2014) The false premises and promises of Bitcoin. arXiv:1312.2048, Cornell University Library

Hayes DG, Gillespie JFE, Daly PH, Grippo G, Johnson PJ (1996) An introduction to digital money issues, prepared for the United States Department of the Treasury Conference "towards digital money and banking: the role of governments", Washington

Houy N (2014) The economics of Bitcoin transaction fees. GATE WP 1407, Groupe d'Analyse et de Théorie Economique, Lyon

Johansen S, Juselius K (1990) Maximum likelihood estimation and inference on cointegration with applications to the demand for money. Oxf Bull Econ Stat 52(2):169-210

Kovenock D, De Vries CG (2002) Fiat exchange in finite economies. Econ Inq 40(2):147-157

Kristoufek L (2013) BitCoin meets google trends and wikipedia: quantifying the relationship between phenomena of the internet era. Sci Rep 3(3415):1-7

Kroll J, Davey I, Felten E (2013) The economics of BitCoin mining, or BitCoin in the presence of adversaries. WEIS 2013. http://weis2013.econinfosec.org/papers/KrollDaveyFeltenWEIS2013.pdf

Krugman PR, Obstfeld M (2003) International economics: theory and policy, 6th edn. Addison Wesley, Boston

Lee TB (2014) These four charts suggest that BitCoin will stabilize in the future. Washington Post. http:// www.washingtonpost.com/blogs/the-switch/wp/2014/02/03/these-four-charts-suggest-that-bitcoinwill-stabilize-in-the-future/

Luther WJ, White LH (2014) Can Bitcoin become a major currency?" GMU working paper in economics No. 14-17, George Mason University

Lütkepohl H, Krätzig M (2004) Applied time series econometrics. Cambridge University Press, Cambridge

Mankiw NG (2007) Macroeconomics, 6th edn. Worth Publishers, New York

Matonis J (2012) Top 10 BitCoin statistics. Forbes 7/31/2012. http://www.forbes.com/sites/jonmatonis/ 2012/07/31/top-10-bitcoin-statistics

Meiklejohn S, Pomarole M, Jordan G, Levchenko K, McCoy D, Voelker GM, Savage S (2013) A fistful of Bitcoins: characterizing payments among men with no names. In: Proceedings of the 2013 ACM internet measurement conference (IMC), pp 127-40. ACM

Moore T, Christin N (2013) Beware the middleman: empirical analysis of BitCoin-exchange risk. Financial Cryptogr Data Secur 7859:25-33

Murphy RP (2013) The economics of BitCoin. Library economic liberty. http://www.econlib.org/library/ Columns/y2013/MurphyBitCoin.html

Nakamoto S (2009) Bitcoin: a peer-to-peer electronic cash system. Bitcoin. http://bitcoin.org/bitcoin.pdf

Palombizio E, Morris I (2012) Forecasting exchange rates using leading economic indicators. Open Access Sci Rep 1(8):1-6

Pantula SG (1989) Testing for unit roots in time series data. Econom Theory 5:256-271

Plassaras NA (2013) Regulating digital currencies: bringing Bitcoin within the reach of the IMF. Chic J Int Law 14(1):377-407

Ron D, Shamir A (2013) Quantitative analysis of the full Bitcoin transaction graph. In: Proceedings of financial cryptography 2013

Satran S (2013) How did Bitcoin become a real currency? U.S. News \& World report. http://money. usnews.com/money/personal-finance/articles/2013/05/15/how-did-bitcoin-become-a-real-currency

Šrda P (2012) Economics of Bitcoin: is Bitcoin an alternative to fiat currencies and gold? Ph.D. thesis, Vienna University of Economics and Business

van Wijk D (2013) What can be expected from the BitCoin?" Working paper no. 345986, Erasmus Rotterdam Universiteit

Velde FR (2013) Bitcoin: a primer. Chicago fed letters no. 317, The Federal Reserve Bank of Chicago

Williams MT (2014) Virtual currencies-Bitcoin risk. Paper presented at the world bank conference, Washington, DC. 21 Oct 2014

Yermack D (2014) Is bitcoin a real currency? An economic appraisal. NBER working paper no. 19747, National Bureau of Economic Research. http://www.nber.org/papers/w19747 